*ak RMIS View/Frint Document Cover Sheet tow

This document was retrieved from the Documentation and Records Manaqement (DRM) ISEARCH System. It is intended for Information only and may not be the most recent or updated version. Contact a Document Service Center (see Hanford Info for locations) if you need additional retrieval information.

Accession \#: D196012497

Document \#: SD-WM-DP-167

Title/Desc:

45 DAY SAFETY SCREENING RESULTS \& FINAL REPORT FOR TANK 241BX106 \& AUGER SAMPLES 95-AUG-049 \& 95-AUG-050

Pages: 55 


\begin{tabular}{|c|c|c|}
\hline $\begin{array}{l}\text { 2. To: (Receiving organization) } \\
\text { Distribution }\end{array}$ & $\begin{array}{l}\text { 3. From: (originating organization) } \\
\text { Characterization Plans, } \\
\text { Coordination and Reports }\end{array}$ & $\begin{array}{r}\text { 4. Related EDT No.: } \\
\text { N/A }\end{array}$ \\
\hline $\begin{array}{l}\text { 5. Proj./Prog./Dept./Div.: } \\
\text { Tank 241-BX-106/Waste } \\
\text { Management/CPCR/Techical } \\
\text { Basis Characterization }\end{array}$ & $\begin{array}{l}\text { 6. Cog. Engr.: } \\
\text { Leela M. Sasaki }\end{array}$ & $\begin{array}{l}\text { 7. Purchase Order No.: } \\
\text { N/A }\end{array}$ \\
\hline \multirow{2}{*}{\multicolumn{2}{|c|}{$\begin{array}{l}\text { 8. originator Remarks: } \\
\text { This document is being released into the supporting document } \\
\text { system for retrievability purposes. }\end{array}$}} & $\begin{array}{l}\text { 9. Equip./Component No.: } \\
\text { N/A }\end{array}$ \\
\hline & & $\begin{array}{c}\text { 10. System/B1dg./Facility: } \\
\text { N/A } \\
\end{array}$ \\
\hline \multirow{3}{*}{\multicolumn{2}{|c|}{$\begin{array}{l}\text { 11. Receiver Remarks: } \\
\text { For release. }\end{array}$}} & $\begin{array}{r}\text { 12. Major Assm. Dwg. No.: } \\
\text { N/A }\end{array}$ \\
\hline & & $\begin{array}{l}\text { 13. Permit/Permit Application No.: } \\
\text { N/A }\end{array}$ \\
\hline & & $\begin{array}{l}\text { 14. Required Response Date: } \\
01 / 22 / 96\end{array}$ \\
\hline
\end{tabular}

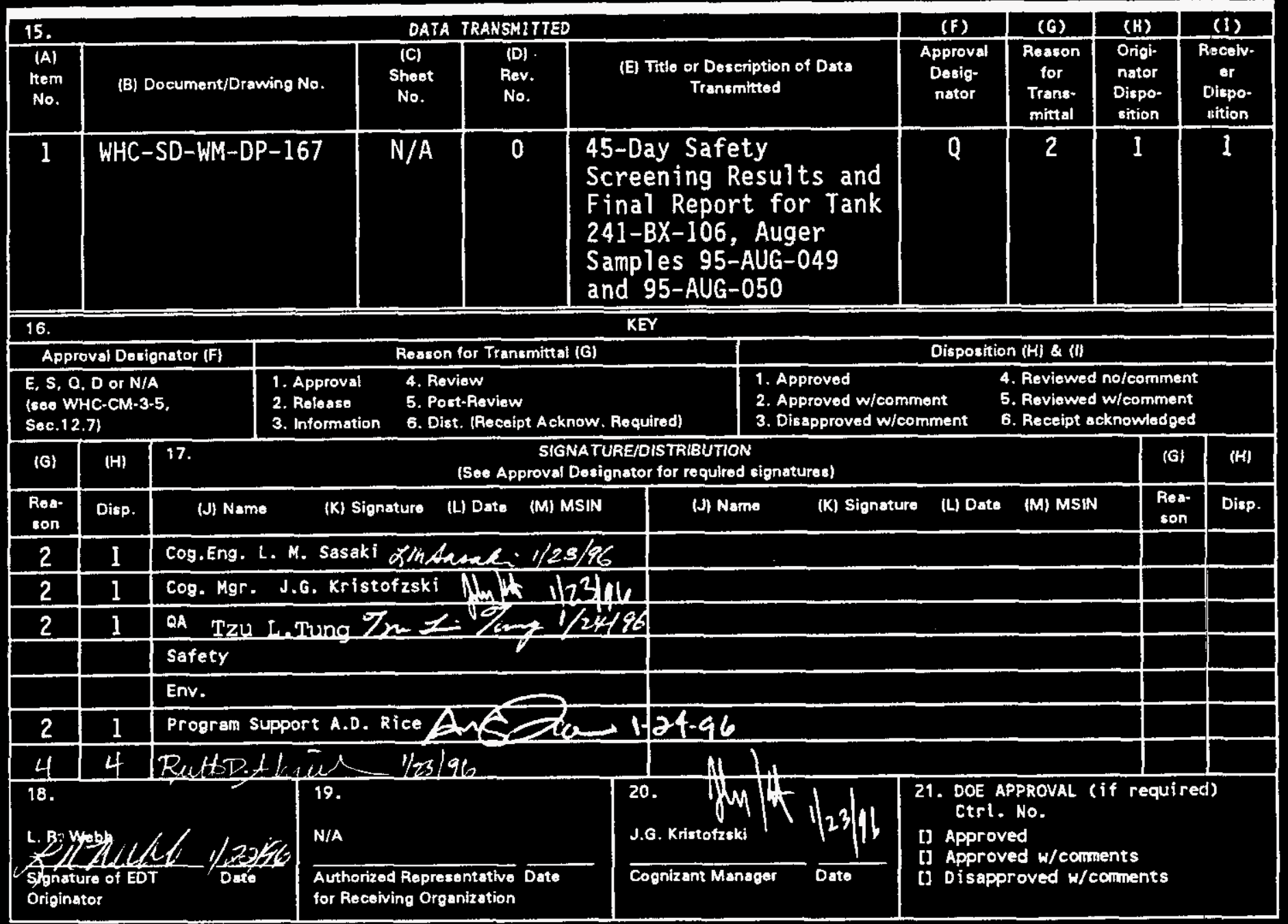




\title{
45-DAY SAFETY SCREENING RESULTS AND FINAL REPORT FOR TANK 241-BX-106, AUGER SAMPLES 95-AUG-049 AND 95-AUG-050.
}

\author{
Leela M. Sasaki \\ Westinghouse Hanford Company, Richland, WA 99352 \\ U.S. Department of Energy Contract DE-AC06-87RL10930

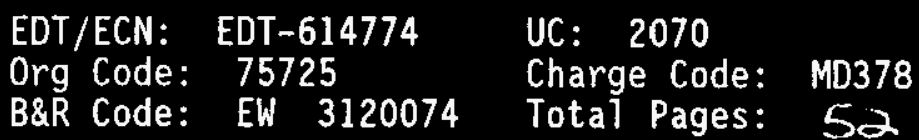

Key Words: 45-Day Safety Screening and Final Results, Auger Samples 95AUG-049 and 95-AUG-050, Tank 241-BX-106 Final Results

Abstract: $N / A$

TRADENARK DISCLAIMER. Reference herein to any specific commercial product, process, or service by trade name, trademark, manufacturer, or otherwise, does not necessarily constitute or imply its endorsement, recormendation, or favoring by the United States Government or any agency thereof or its contractors or subcontractors.

Printed in the United States of America. To obtain copies of this document, contact: WHC/BCS Document Control Services, P.O. Box 1970, Mailstop H6-08, Richland WA 99352, Phone (509) 372-2420; Fax (509) 376-4989.
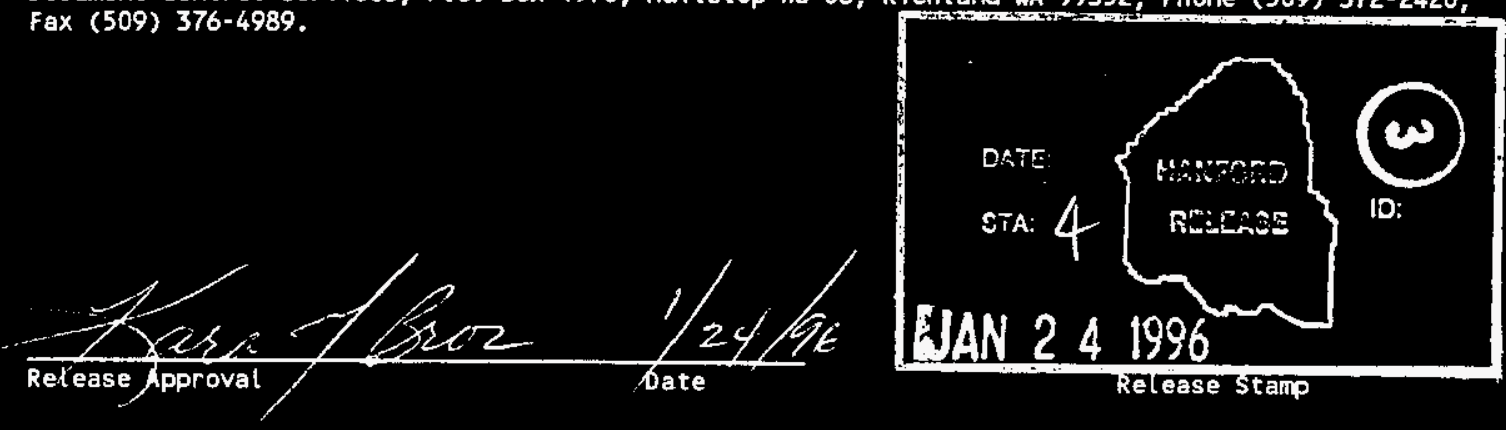

Approved for Public Release 


\section{Q2) Westinghouse

P.O. Box 1970 Richland, WA 99352

WHC-SD-WM-DP-167, REV. 0

ANALYTICAL SERVICES

45-DAY SAFETY SCREENING RESULTS AND FINAL REPORT FOR TANK 241-BX-106, AUGER SAMPLES 95-AUG-049 AND 95-AUG-050

Project Coordinator: Leela M. Sasaki

Prepared for the U.S. Department of Energy

Office of Environmental Restoration and Waste Management

by

Westinghouse Hanford Company

Box 1970

Richland, Washington 
WHC-SD-WH-DP-167, REV. 0

TABLE OF CONTENTS

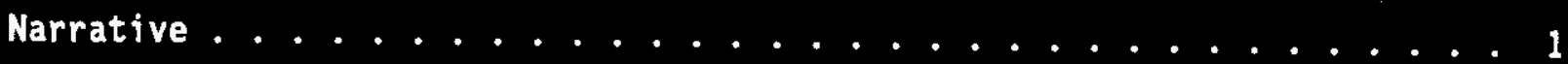

Sample Data Sumary ................... 7

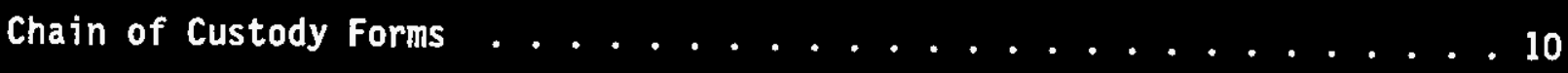

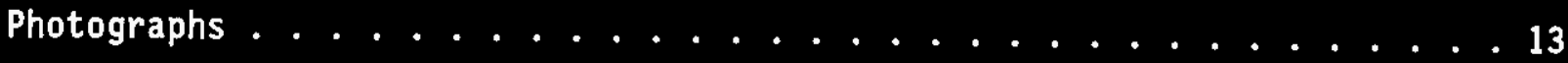

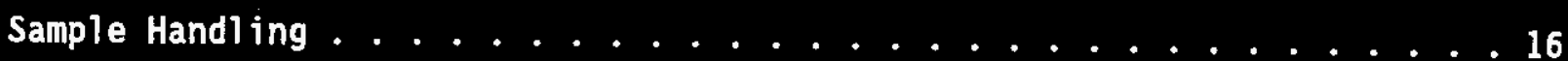

Bulk Density Worksheet . . . . . . . . . . . . 17

Extrusion Worklist \# 4731 (Riser 6 ) . . . . . . . . . 19

Extrusion Workl ist \#4732 (Riser 2) .......... 20

Sample Preparations ..................... 21

Fusion Digestion Work1 ist \#4777 ............ 22

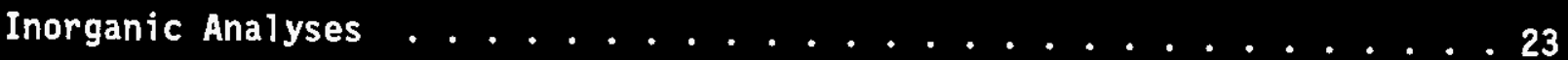

Differential Scanning Calorimetry (DSC)

DSC Worklist \# 4849 .................... . 24

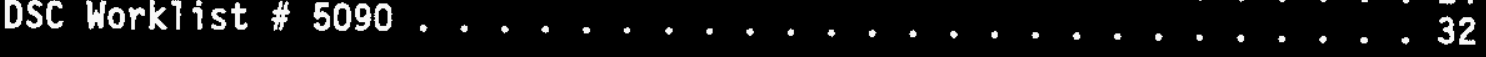

Thermogravimetric Analysis (TGA)

TGA Work ist \#4847.................. 33

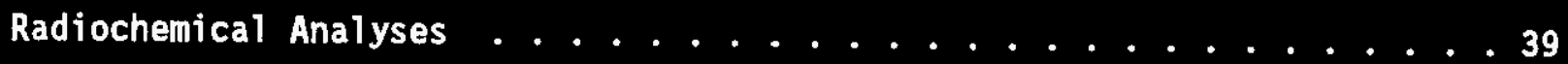

Total Alpha Analysis (AT)

AT Work 1 ist \#4862 ................... . 40

This Document consists of pages 1 through 49 . 
WHC-SD-WM-DP-167, REV. 0

NARRATIVE 


$$
\text { WHC-SD-WM-DP-167, REV. } 0
$$

\section{SUMMARY}

Two auger samples from tank 241-BX-106 (BX-106) were received at the 222-S Laboratory and underwent safety screening analyses - differential scanning calorimetry (DSC), thermogravimetric analysis (TGA), total alpha analysis, and bulk density measurements - in accordance with the sampling and analys is plan (SAP) (Sasaki 1995).

No safety screening notification limits were exceeded on any of the BX-106 samples. No exotherms were observed and the upper 1 imits of the one-sided 95percent confidence intervals for total alpha activity results were well below the notification limit. Tank headspace flammability was measured at 0 percent of the lower flammability limit (LFL).

\section{SCOPE}

This document serves as the 45-day and final report deliverable for the tank BX-106 auger samples (95-AUG-49 and 95-AUG-50) collected between December 15 and December 18, 1995. The samples were received, extruded, and analyzed by the 222-S Laboratory in accordance with the referenced SAP (Sasaki 1995). Any exceptions to the plan are discussed in the following sections.

This report includes the safety screening results (tank headspace flammability, DSC, TGA, total alpha activity, and bulk density) and copies of all DSC and TGA raw data scans as requested per the SAP. As the final report, the following are also included: chains of custody; bulk density worksheets; sample preparation data; and total alpha analysis raw data.

\section{SAMPLE RECEIPT AND EXTRUSION}

Auger Sample 95-AUG-49, Riser 6

Auger sample 95-AUG-49 was collected from riser 6 of tank BX-106 on December 15,1995 using a 20-inch auger bit with flutes 1 inch apart. The auger sample was received at the 222-S Laboratory on December 22, 1995 and extruded on January 5, 1996. The sample was contained on the bottom 8 flutes of the auger sampler. The solids were black in color and the texture was that of a soft, wet sludge. The sample also contained about three small pieces of white material. A total of 63.1 grams of solids were recovered from the sampler. No drainable liquids were present in the sample. Sample numbers associated with this auger sample are: S96T000110, S96T000112, and S96T000113. Details of the extrusion and subsampling are provided in the hot cell logbook for tank BX-106 (Fuller 1995).

\section{Auger Sample 95-AUG-50, Riser 2}

Auger sample 95-AUG-50 was collected from riser 6 of tank BX-106 on December 18 , 1995 using a 20-inch auger bit with flutes $1 / 2$ inch apart. The auger 
WHC-SD-WM-DP-167, REV. 0

sample was received at the 222-S Laboratory on December 22, 1995 and extruded on January 5, 1996. The sample was contained on the bottom 15 flutes of the auger sampler. The solids were black in color and the texture was that of a soft, wet sludge. A total of $\mathbf{4 0 . 9}$ grams of solids were recovered from the sampler. No drainable liquids were present in the sample. The sample also contained an electrical cord which was wrapped around the auger and across several flutes; the cord was removed and set aside. Sample numbers associated with this auger sample are: S96T000114, S96T000116, and S96T000117. Details of the extrusion and subsampling are provided in the hot cell logbook for tank BX-106 (Fuller 1995).

\section{TANK HEADSPACE FLAMMABILITY}

Prior to core sampling, the flammability of the tank headspace was measured to address the vapor flammability issue of the safety screening data quality objective (Dukelow, et al. 1995). The tank vapor space was sampled on December 20, 1995 in accordance with work package ES-95-00586 to determine flammability of the vapor space gasses. Sampling of the tank dome space was done through riser 2 . The combustible gas meter reading was 0 percent of the LFL, indicating no flammability concerns with this tank. Other measurements made were oxygen (20.9 percent), total organic vapors ( 1.5 parts per million), and ammonia ( 2 parts per million).

\section{ANALYTICAL RESULTS}

Analytical results appear in the Sample Data Summary and a discussion of the results is presented below. Any deviations from the requirements of the SAP are noted in the following sections.

\section{TGA (Moisture)}

The weight percent water by TGA was performed under a nitrogen purge using procedure LA-560-112, Rev. B-1 on a Mettler TG 50 instrument.

The average result for auger sample 95-AUG-49 (sample S96T000112) was 36.20 percent with a relative percent difference (RPD) of 19.0 percent. The average result for auger sample 95-AUG-50 (sample S96T000116) was 41.18 percent with a RPD of 11.1 percent. Relative percent differences for the two samples were outside the \pm 10 percent required by the SAP; reruns were not requested because the TGA results are only needed to calculate the exotherms on a dry weight basis and no exotherms were exhibited by the samples. The standard recovery was 102.9 percent, within the required 90 to 110 percent range. 
WHC-SD-WM-DP-167, REV. 0

$\underline{\mathrm{DSC}}$

Differential scanning calorimetry analyses were performed under a nitrogen purge using procedure LA-514-114, Rev. C-1 on a Perkin Elmer DSC 7 instrument. The DSC scans from the Perkin Elmer instrument show exotherms as valleys and endotherms as peaks. Using the average percent water by TGA for each sample, exotherms in $\mathrm{J} / \mathrm{g}$ on a dry weight basis are calculated from the exotherms in $\mathrm{J} / \mathrm{g}$ on a wet weight basis.

No exotherms were observed in either of the waste samples. Standards recoveries were 100.4 percent and 96.34 percent, within the required range of 90 to 100 percent.

\section{Total Alpha Activity}

The total alpha analyses were performed using procedure LA-508-101, Rev. D-2. The solid samples were prepared by fusion prior to the total alpha analysis; the fusion was performed using procedure LA-549-141, Rev. D-0.

The SAP specifies a total alpha notification limit of $41 \mu \mathrm{Ci} / \mathrm{g}$. This number is derived from the safety screening data quality objective's notification $1 \mathrm{imit}$ of $1 \mathrm{~g} / \mathrm{L}$ of plutonium using the conservative assumptions that all the plutonium is plutonium-239 and the density is $1.5 \mathrm{~g} / \mathrm{mL}$. If the actual density of a sample is above $1.5 \mathrm{~g} / \mathrm{mL}$, the notification 1 imit of $41 \mu \mathrm{Ci} / \mathrm{g}$ was to be recalculated using the higher density number (Sasaki 1995). The highest measured density for the BX-106 auger samples was $1.68 \mathrm{~g} / \mathrm{mL}$; using this result, the notification limit would be $36.6 \mu \mathrm{Cj} / \mathrm{g}$.

The average results for the samples were $0.619 \mu \mathrm{Ci} / \mathrm{g}$ and $0.560 \mu \mathrm{Ci} / \mathrm{g}$ for auger sample 95-AUG-49 (sample S96T000113) and auger sample 95-AUG-50 (sample S96T000117), respectively. The RPDs for 95-AUG-49 and 95-AUG-50 were within the required \pm 20 percent at 10.7 percent and 6.07 percent, respectively. The standard recovery for both samples was 90.26 percent, within the 80 to 120 percent range required by the SAP. Spike recoveries were slightly outside the required 80 to 120 percent range at 79.59 and 79.08 percent. No reruns were requested since the total alpha results were well below the notification limit.

The one-sided 95-percent upper confidence interval was calculated for the two auger samples. The upper $1 \mathrm{imit}$ of the one-sided 95-percent confidence interval was $0.827 \mu \mathrm{Ci} / \mathrm{g}$ for 95-AUG-49 (sample S96T000113) and $0.667 \mu \mathrm{Ci} / \mathrm{g}$ for 95-AUG-50 (sample S96T000117), both well below the notification 1 imit of 36.6 $\mu \mathrm{Ci} / \mathrm{g}$.

\section{Bulk Density}

Bulk density measurements were performed on the samples using procedure L0-160-103, Rev. A-7. 


\section{WHC-SD-WM-DP-167, REV. 0}

Bulk densities of the solids were $1.68 \mathrm{~g} / \mathrm{mL}$ for auger sample 95-AUG-49 (sample S96T000113) and $1.60 \mathrm{~g} / \mathrm{mL}$ for auger sample 95-AUG-50 (sample S96T000117). Due to insufficient amounts of sample, no duplicate bulk density measurements were performed.

Project Coordinator: L. M. Sasaki 


\section{WHC-SD-WM-DP-167, REV. 0}

\section{REFERENCES}

Dukelow, G. T., J. W. Hunt, H. Babad, and J. E. Meacham, 1995, Tank Safety Screening Data Quality Objective, WHC-SD-WM-SP-004, Rev. 2, Westinghouse Hanford Company, Richland, Washington.

Fuller, R. K., 1995, Tank BX-106, WHC-N-1138, Westinghouse Hanford Company, Richland, Washington.

Sasaki, L. M., 1995, Tank 241-BX-106 Auger Sampling and Analysis Plan, WHC-SD-WM-TSAP-068, Rev. 1, West inghouse Hanford Company, Richland, Washington. 
WHC-SD-WM-DP-167, REV. 0

SAMPLE DATA SUMMARY 


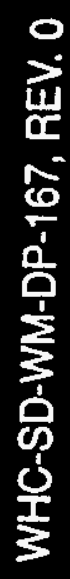

7im! 7 pajajas < pajejo!n t! u! $7<$

\begin{tabular}{|c|c|c|c|c|c|c|c|c|c|c|c|c|c|c|}
\hline $10+367 i$ & $20-550 \cdot 9$ & $65^{\circ} 62$ & $\angle \mathrm{OL}$ & $10-36 L^{\circ 9}$ & $10-398^{\circ} 5$ & $10.225^{\circ} 9$ & $20-2\left\langle 0^{\circ} \eta\right\rangle$ & $92^{\prime} 06$ & (3) & $00+20^{\circ} 1-$ & $5 / 13 n$ & p!los pajsasta to eqdiv & 」 & El $000196 \mathrm{~S}$ \\
\hline$\frac{e / 0}{e / 4}$ & $e / u$ & E/u & 000 & $00+2000$ & $00+2000$ & $00+300 \cdot 0$ & $\mathrm{e} / \mathrm{u}$ & $\mathrm{e} / \mathbf{u}$ & 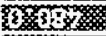 & $00+20 \div 1-$ & XJa b/sajnop & 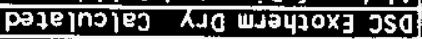 & & 210001965 \\
\hline e/u & e/u & e/u & $00^{\circ} 0$ & $00+200^{\circ} 0$ & $00+2000$ & $00+300^{\circ} 0$ & $e / u$ & 7.001 & 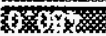 & $00+201-$ & 6/sopinor & 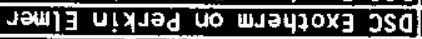 & & Z110001965 \\
\hline$\frac{E / u}{B / M}$ & $\mathrm{E} / \mathrm{u}$ & $\mathrm{E} / \mathrm{u}$ & 0.61 & $029 \varepsilon$ & $S L Z \Sigma$ & $79^{\circ} 6 \varepsilon$ & e/u & 6.201 & काण & गUणN & $\bar{z}$ & 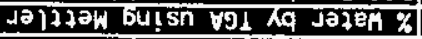 & & $211000196 \mathrm{~S}$ \\
\hline $\mathrm{E} / \mathrm{u}$ & $10-200^{\prime} \mathrm{S}$ & $\mathrm{E} / \mathbf{U}$ & e/u & e/u & e/u & $089^{\prime \prime} \mathrm{L}$ & $e / 0$ & E/u & כuON & DUON & 7 पण & ajcues to $x$ ?! suag $\times 1 n$ g & & $0 \mathrm{~b} 1000196 \mathrm{~S}$ \\
\hline XdJG JUNOJ & $7 ! 0 ! 7200$ & \% כग्य ॠas & $\%$ 0dy & ВОЕJקAY & 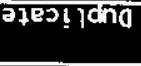 & tinsey & त्रue 18 & \% pJepuezs & 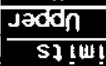 & \begin{tabular}{|l|} 
Jамо 7 \\
\end{tabular} & z!un & 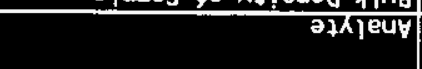 & $\# \forall$ & 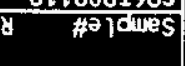 \\
\hline
\end{tabular}

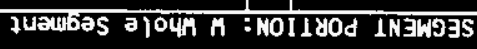

67-9n日-56 :\# 1N3w93S

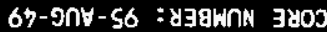

(v) $901-x 8$

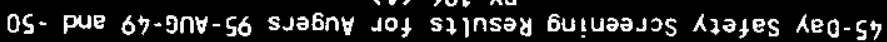




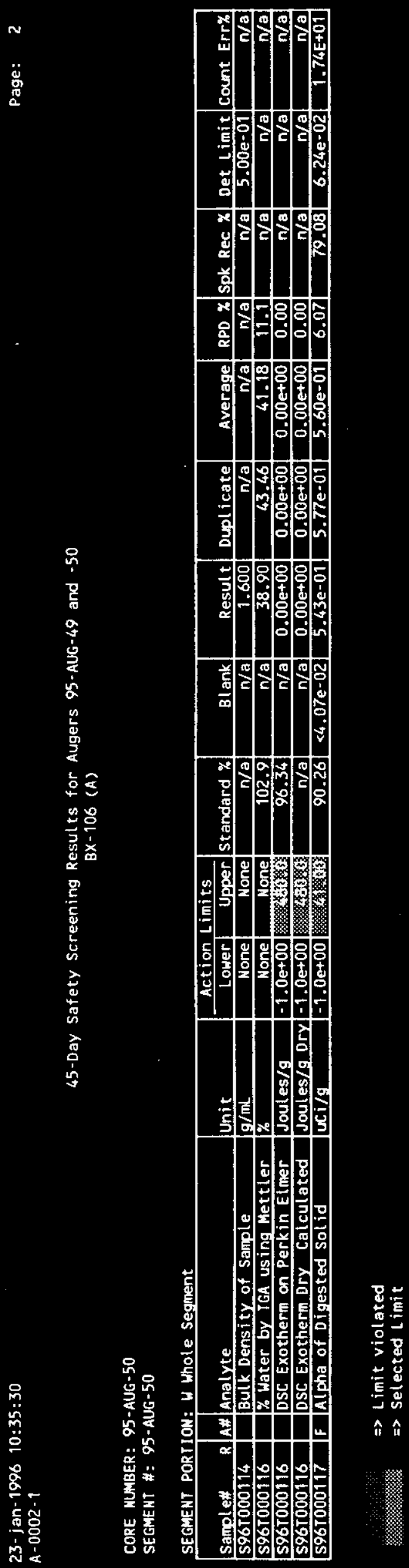

WHC-SD-WM-DP-167, REV. 0 
WHC-SD-WM-DP-167, REV. 0

CHAIN OF CUSTODY FORMS 







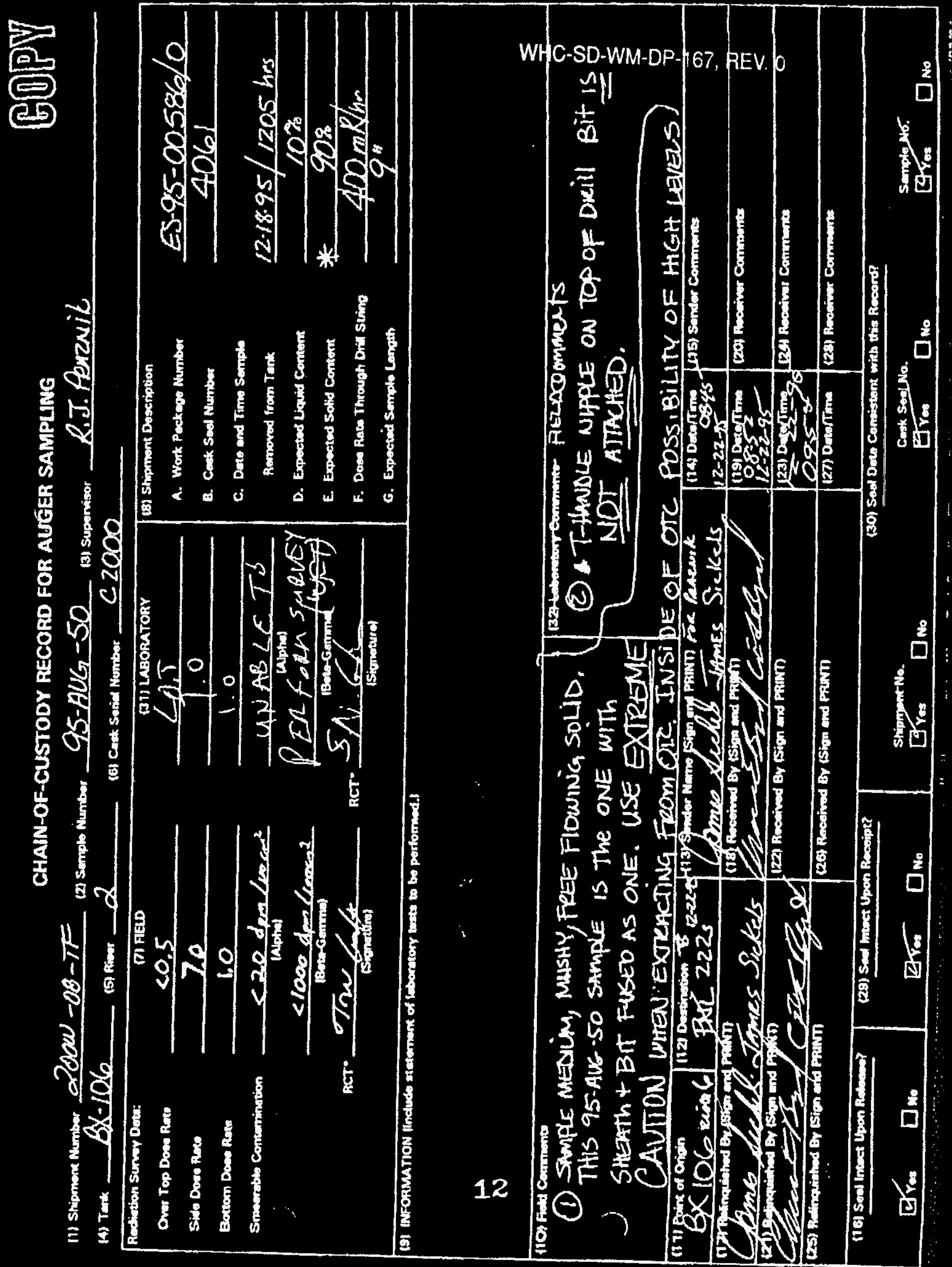


WHC-SD-WM-DP-167, REV. 0

PHOTOGRAPHS 


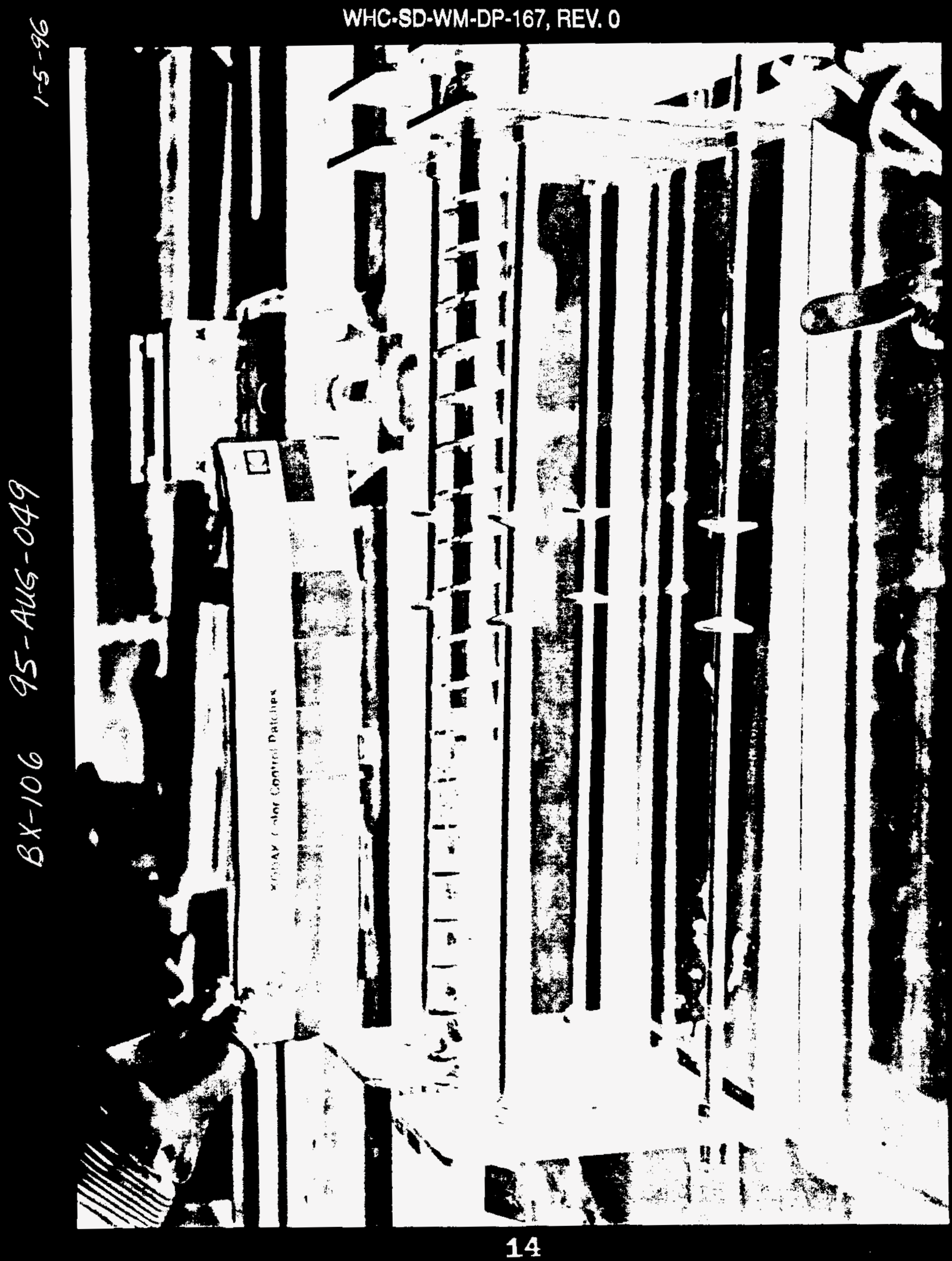




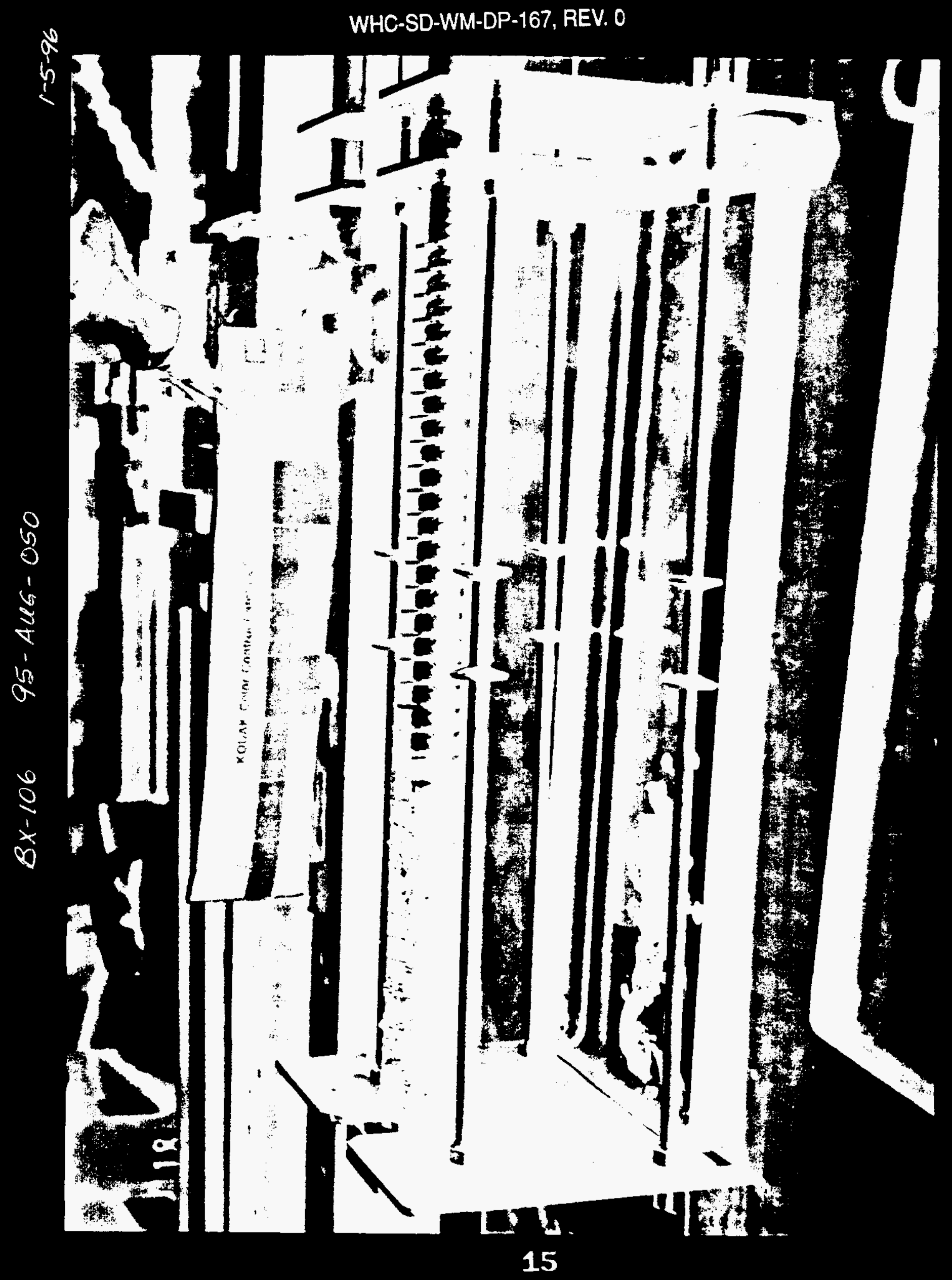


WHC-SD-WM-DP-167, REV. 0

SAMPLE HANDLING 


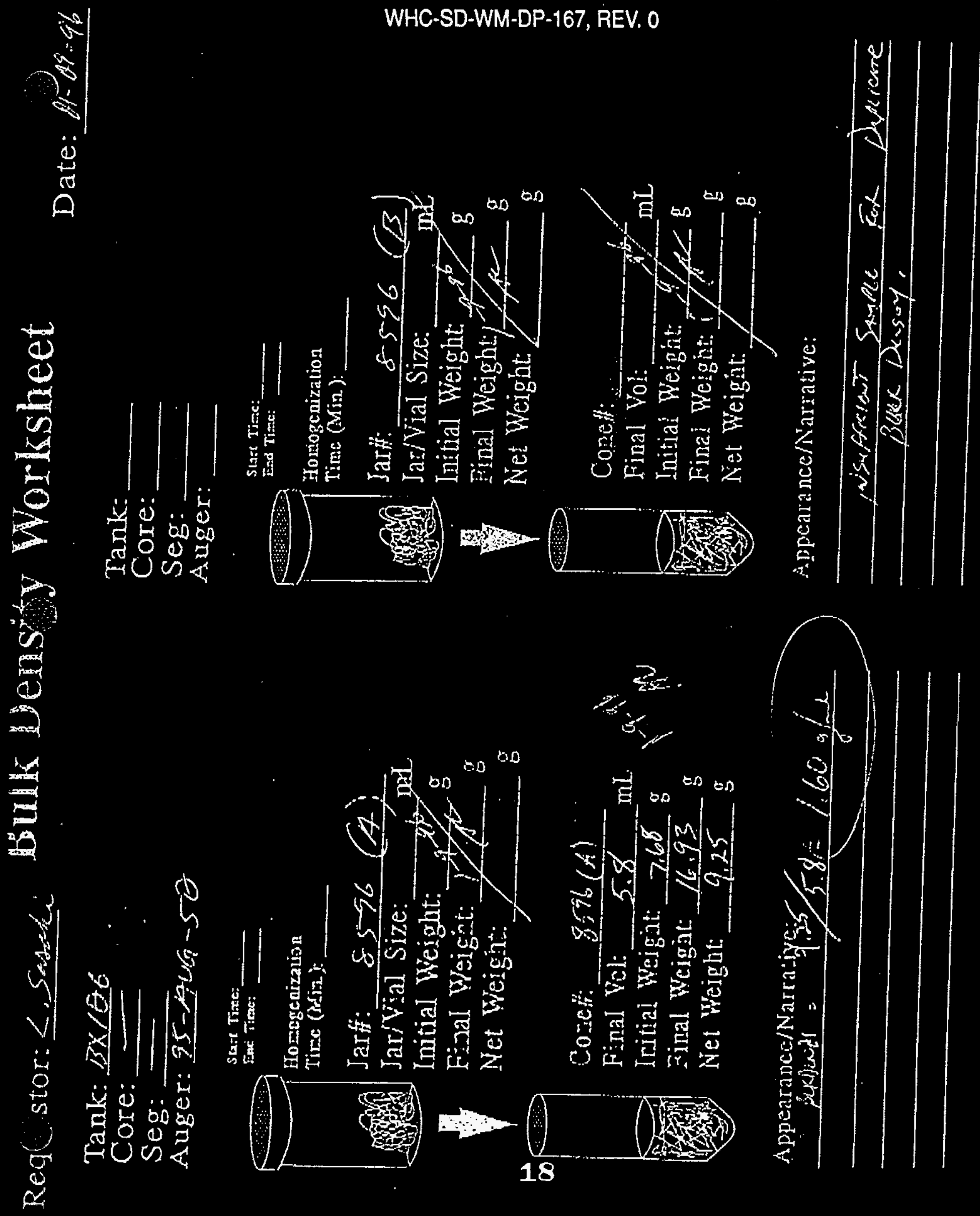




\section{LABCORE Data Entry Template for Worklist:}

\section{Analyst: \\ Instrument: BA000 \\ Method: LO-160-103 Rev/Mod A-7 \\ Worklist Comment: BX-106 95-AUG-49 RISER 6 EXTRUSION}

Book \#

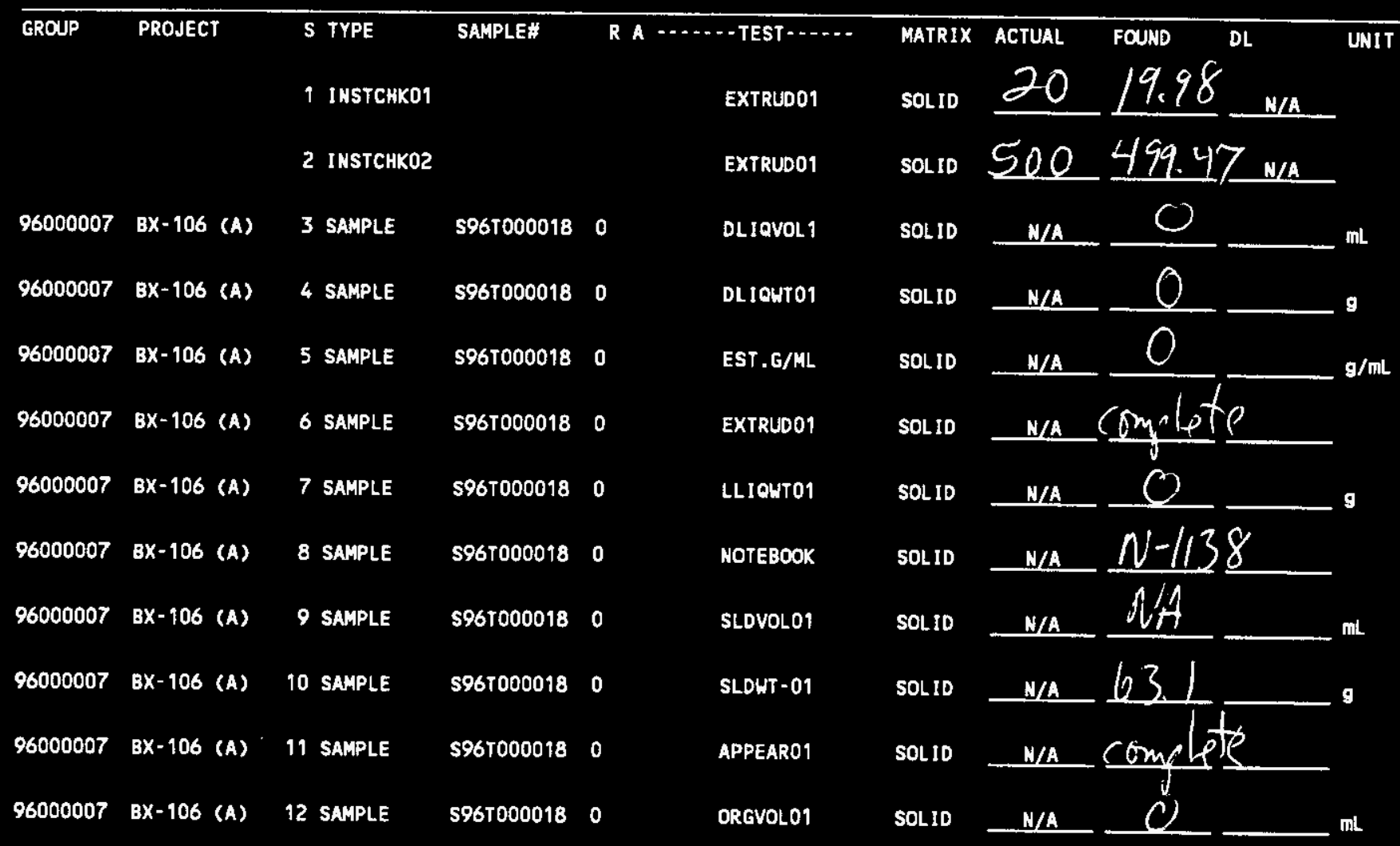

\section{Final page for worklist \# 4731}

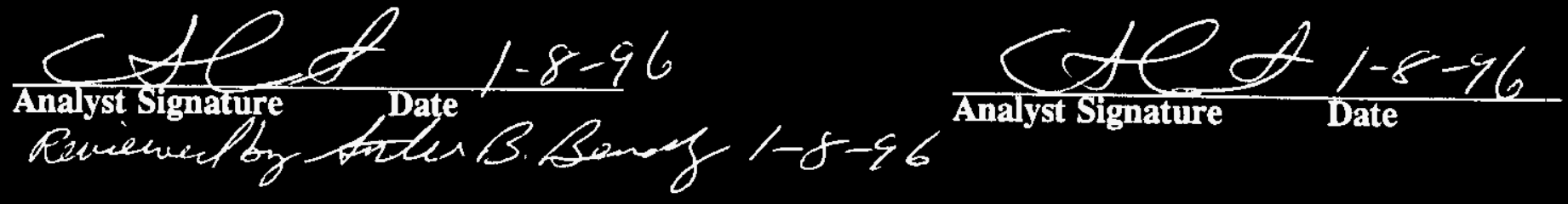

Data Entry Comments:

Units shown for $Q C$ (SPK \& STD) may not reflect the actual units. $D L=$ Detection Limit, $S=$ Worklist Slot Number, $R=$ Replicate Number, $A=$ Aliquot Code. 


\section{Analyst: $\frac{C(C}{\text { Instrument: BA000 }}$
Method: LO-160-103 Rev/Mod $A-7$
Worklist Comment: BX-106 95-AUG-50 RISER 2 EXTRUSION}

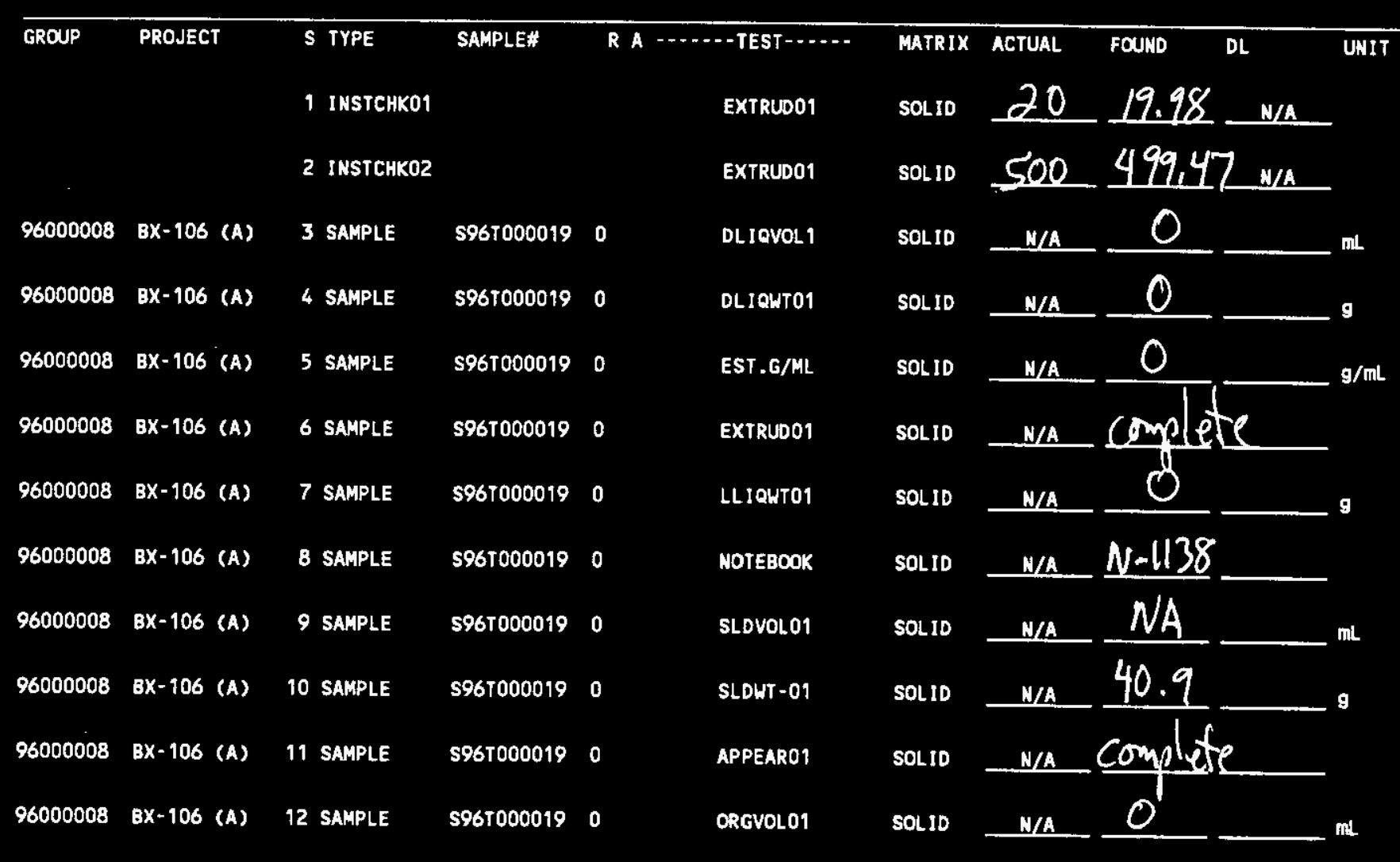

\section{Final page for worklist \# 4732} Book \# NA

\section{Book}

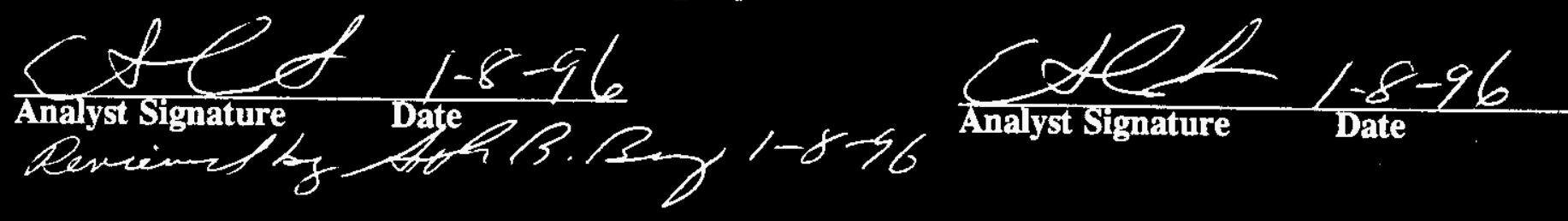

Data Entry Comments:

Units shown for $Q C$ (SPK \& STD) may not reflect the actual units. $D L=$ Detection Limit, $S=$ Worklist Slot Number, $R=$ Replicate Number, $A=$ Aliquot Code. 
WHC-SD-WM-DP-167, REV. 0

SAMPLE PREPARATIONS

21 


\section{LABCORE Data Entry Template for Worklist}

Analysti GXM Instrument: Fus01 SNl6355 Bonk"

Method: LA-549-141 Rev/Mod $\varepsilon-\mathcal{O}$

Worklist Comment: BX-106 95-^UG-049 \& 95-AUG-050 Fusion Digestion rkt

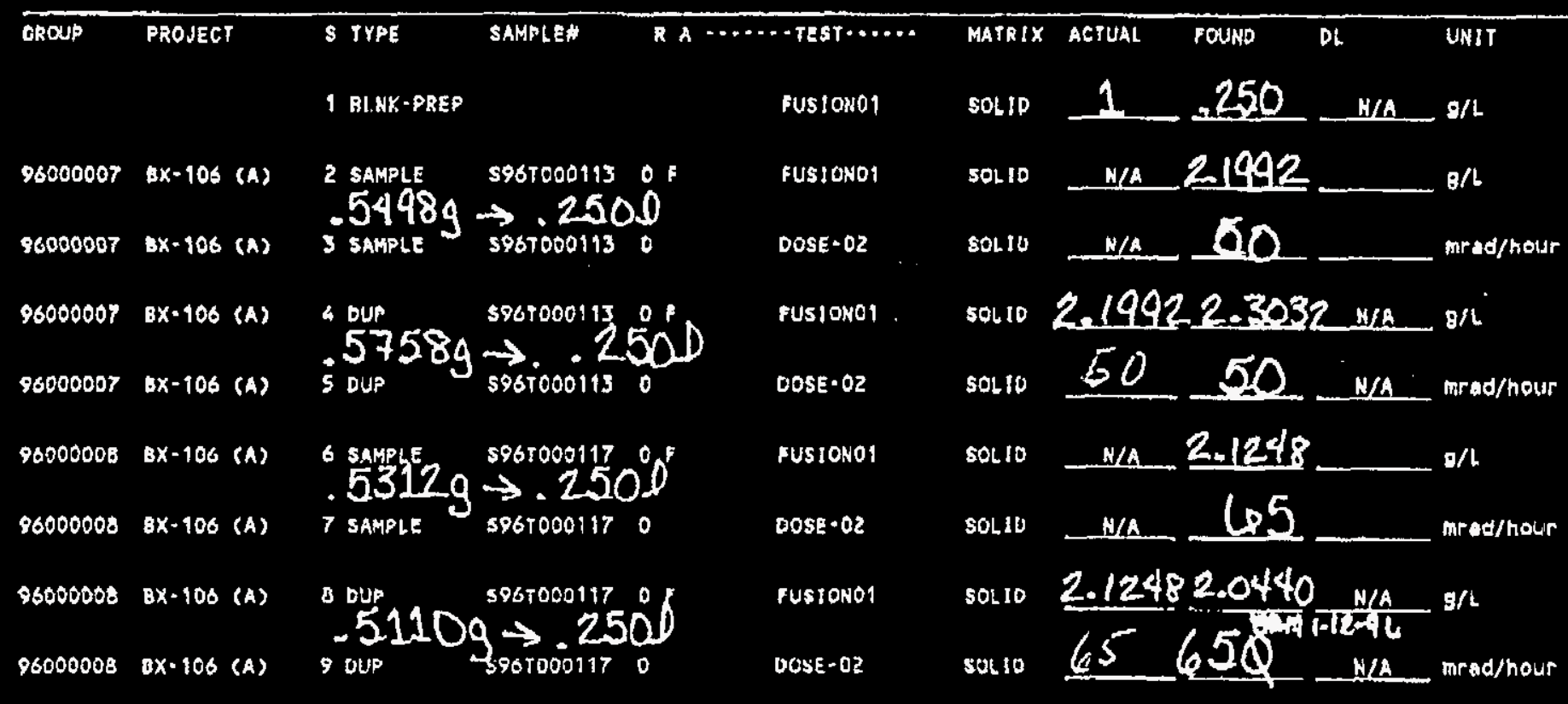

\section{Final page for worklist \# 4777}

MNMunhy $\frac{1-10-96}{\text { Date }}$

$\begin{array}{lll}546+000112 & \rightarrow 3 & 113 \\ 5967000116 \rightarrow-\cdots & 117\end{array}$

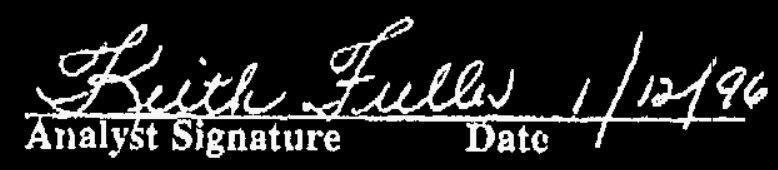

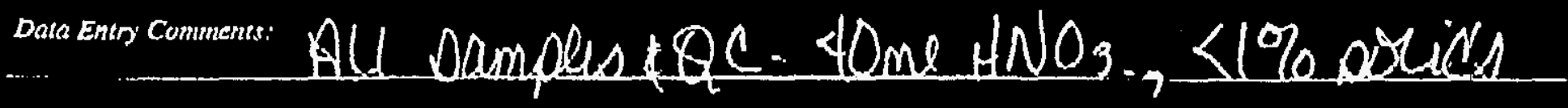

Units shown for $Q C$ (SPK \& STD) muy not reflect the actual units. $D L=$ Delectlon Limit, $S=$ Worklist Slot Number, $R=$ Replicute Number, $A=$ Aliguor Code. 
WHC-SD-WM-DP-167, REV. 0

INORGANIC ANALYSES

23 


\section{LABCORE Data Entry Template for Worklist\#}

Analyst: $\quad$ ADP $\quad$ Instrument: DSC0 3 Book \# $\underline{12 N 14 B}$

Method: LA-514-114 Rev/Mod $\frac{C-1}{D_{s e s}}$

Worklist Comment: Please run BX-106 Des under N2. bdv

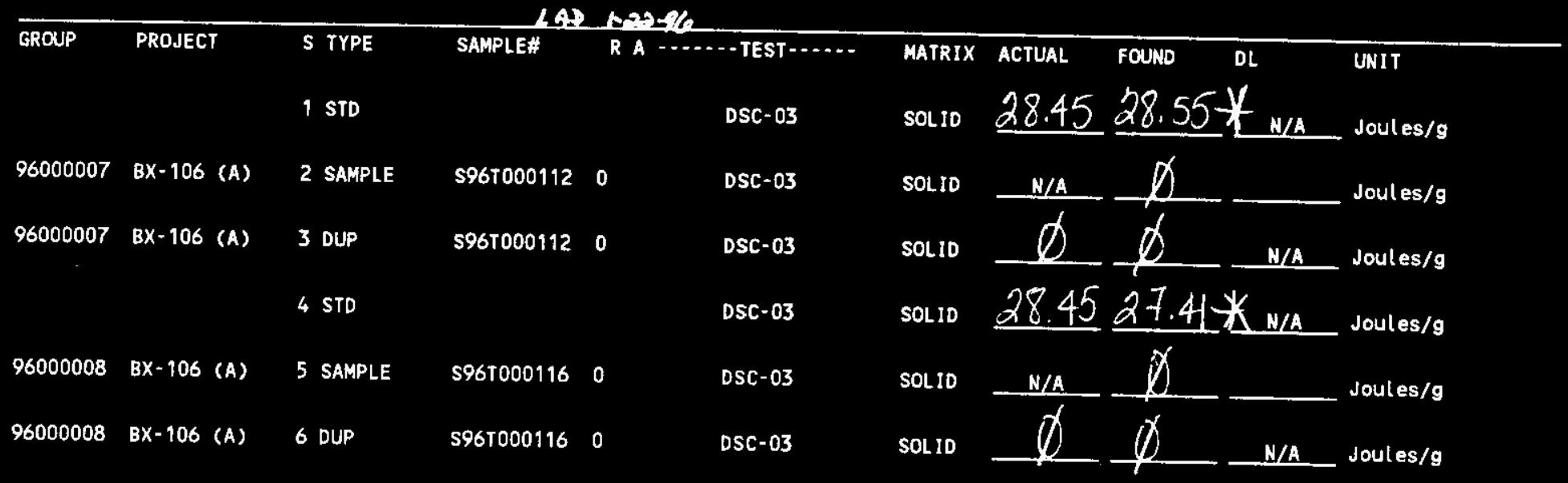

\section{$\int_{\text {nalyst Signature }} \frac{\text { Final page }}{\text { Date }}$}

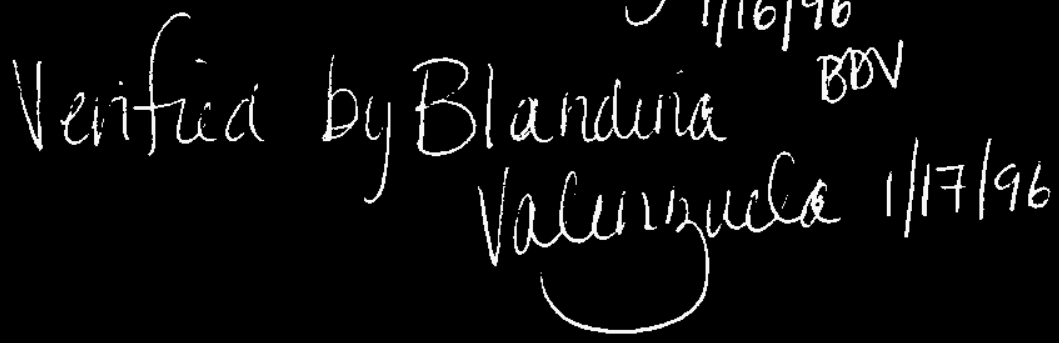

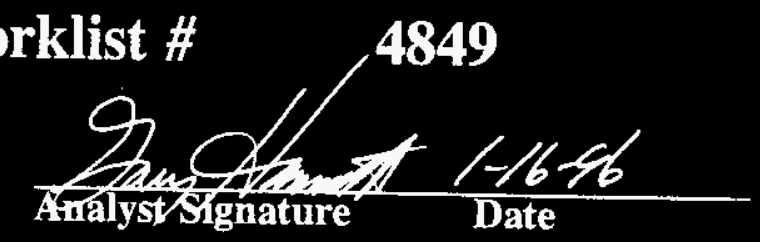

$\|\left. 17\right|_{\text {bit }}$

S96TOCOI12 produced at three endotheroms one at $130.53^{\circ} \mathrm{C}$

with a dieta $H$ of $671.82 \mathrm{~J} / \mathrm{g}$, Decond at $278.3^{\circ} \mathrm{C}$ with a deita $\mathrm{H}$ i. $404.3 \mathrm{~J}$

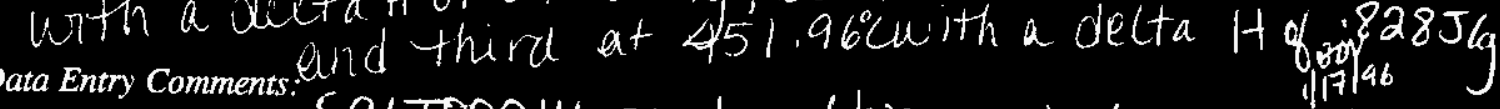

5967000116 produced two endothems ed one at $131.96^{\circ} \mathrm{C}$ with a delta $H$ of $811.57 \mathrm{Jlg}$ and second at $315.84^{\circ} \mathrm{C}$ with a delta H of $270.7 \mathrm{~J} / \mathrm{g}$

Units shown for $Q C$ (SPK \& STD) may not reflect the actual units. $D L=$ Detection Limit, $S=$ Worklist Slot Number,

$R=$ Replicate Number, $A=$ Aliquot Code. 


\section{LABCORE Data Entry Template for Worklist\#}

Analyst: ADP Instrument: DSCO Book $12 N 14 \mathrm{~B}$

Method: LA-514-113 Rev/Mod $C-1$

Worklist Comment: Please run BX-106 fis under N2. bdv

\begin{tabular}{|c|c|c|c|c|c|c|c|c|c|c|}
\hline \multirow[t]{2}{*}{ GROUP } & PROJECT & $S$ TYPE & SAMPLE\# & R A & - & MATRIX & ACTUAL & FOUND & DL & UNIT \\
\hline & & 1 STD & & & DSC-01 & SOLID & & & N/A & Joules/g \\
\hline 96000007 & $B X-106$ (A) & 2 SAMPLE & s96r000112 & 0 & DSC-01 & SOLID & N/A & & & Joules/9 \\
\hline 96000007 & $B X-106(A)$ & 3 DUP & S96T000112 & 0 & DSC-01 & SOL.ID & & & $\mathbf{N} / \mathbf{A}$ & Joules/g \\
\hline 96000008 & $B \times-106$ (A) & 4 SAMPLE & S96T000116 & 0 & DSC-01 & SOLID & $N / A$ & & & Joules/9 \\
\hline 96000008 & BX-106 (A) & 5 DUP & S96T000116 & 0 & DSC-01 & SOLID & & & $\mathrm{N} / \mathrm{A}$ & Joules/g \\
\hline
\end{tabular}

\section{Final page for worklist \# 4849}

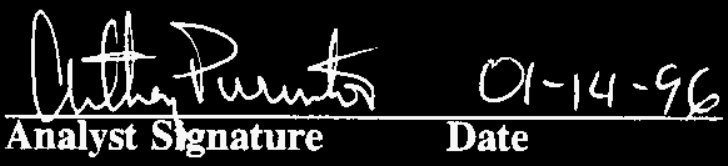

Analyst Signature Date

Data Entry Comments:

Units shown for QC (SPK \& STD) may not reflect the actual units. $D L=$ Detection Limit, $S=$ Worklist Slot Number, $R=$ Replicate Number, $A=$ Aliquot Code. 


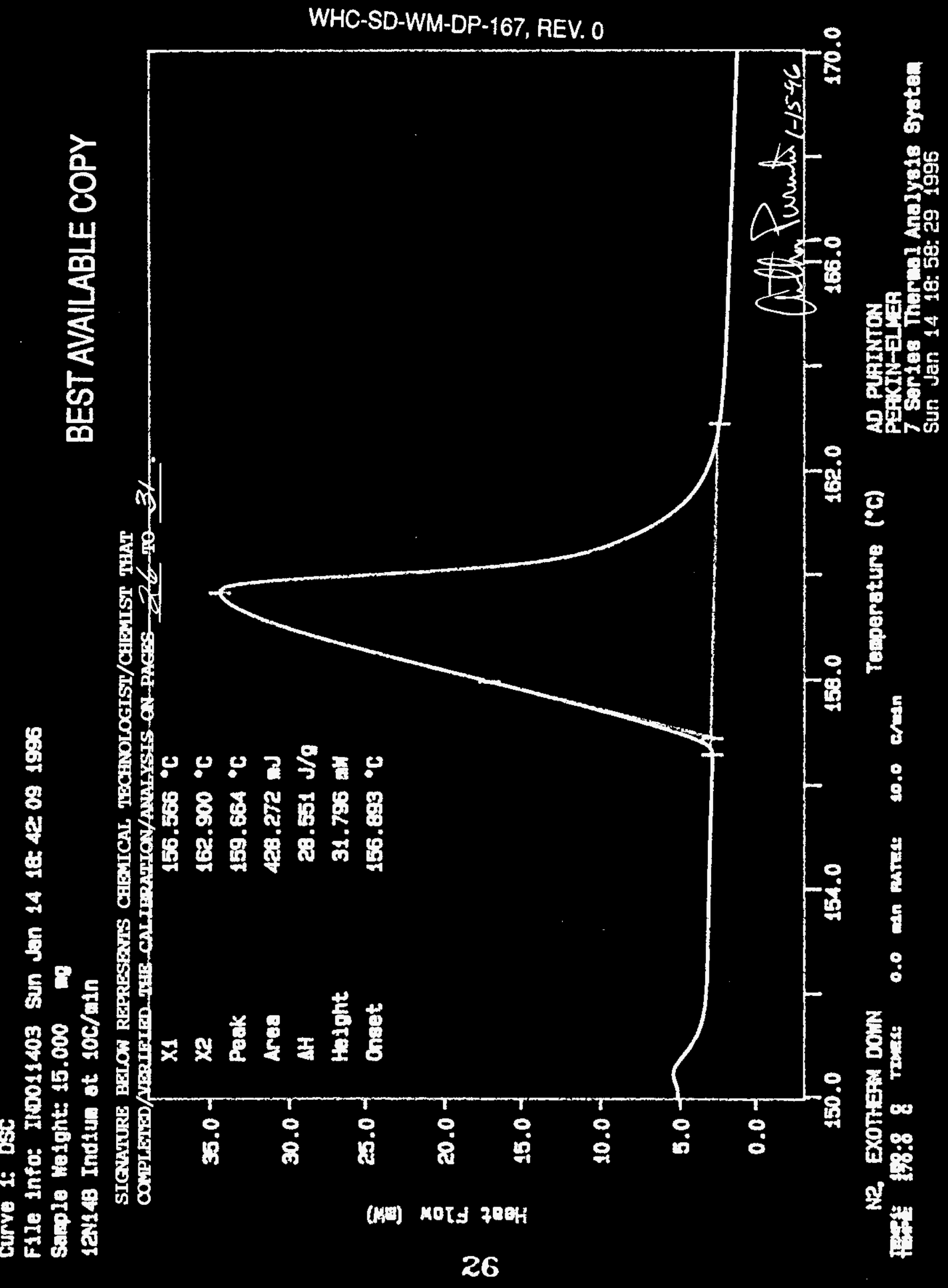




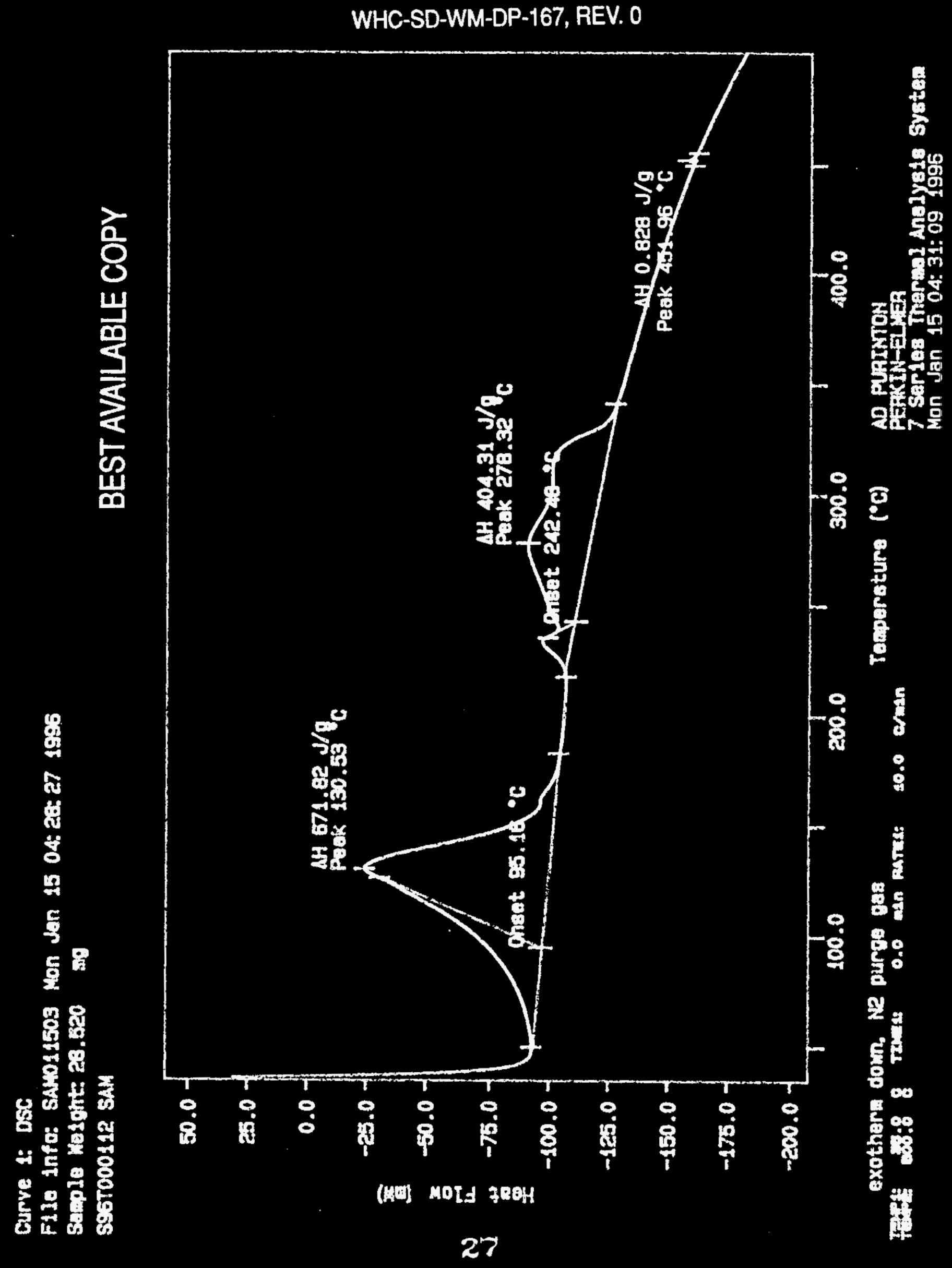




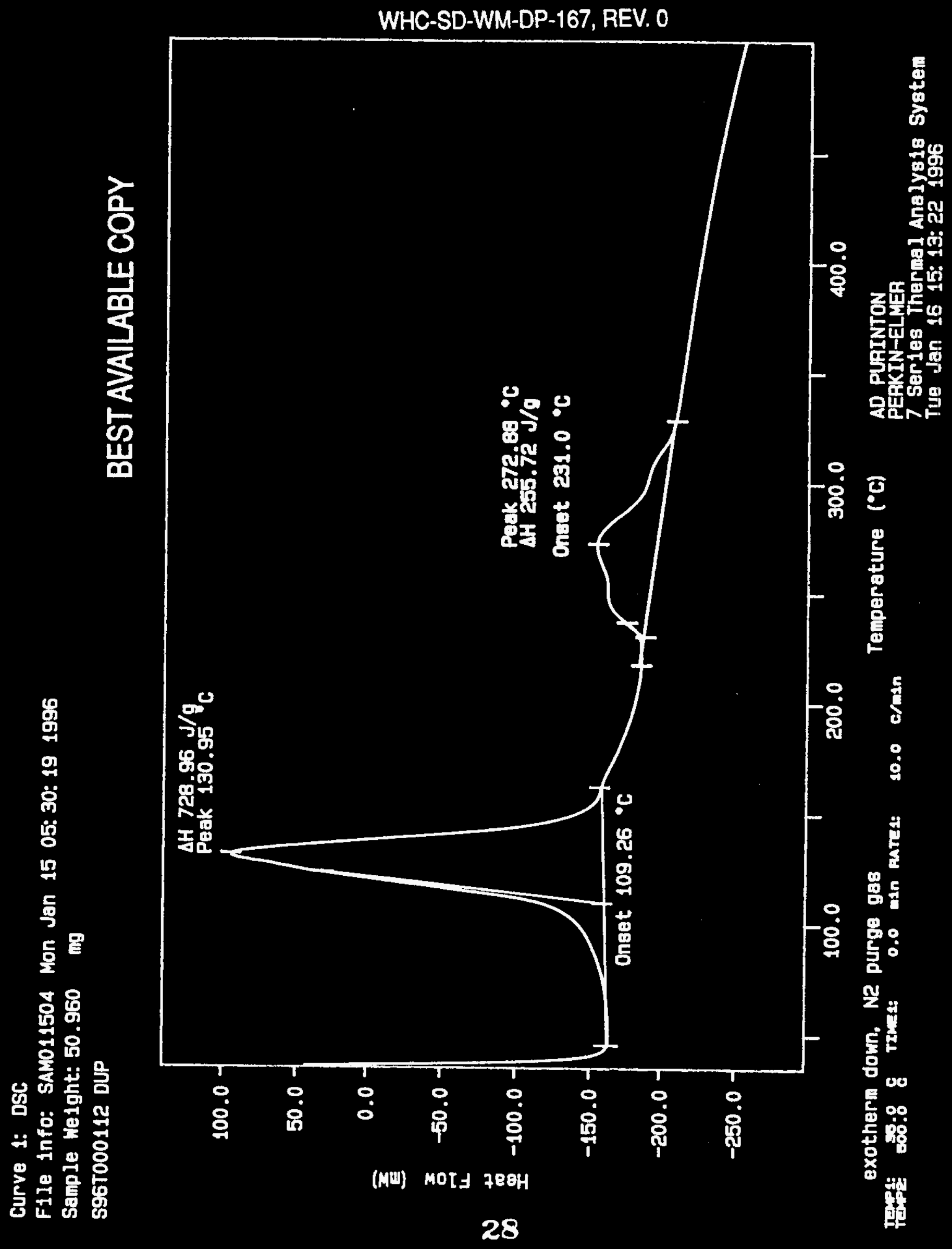




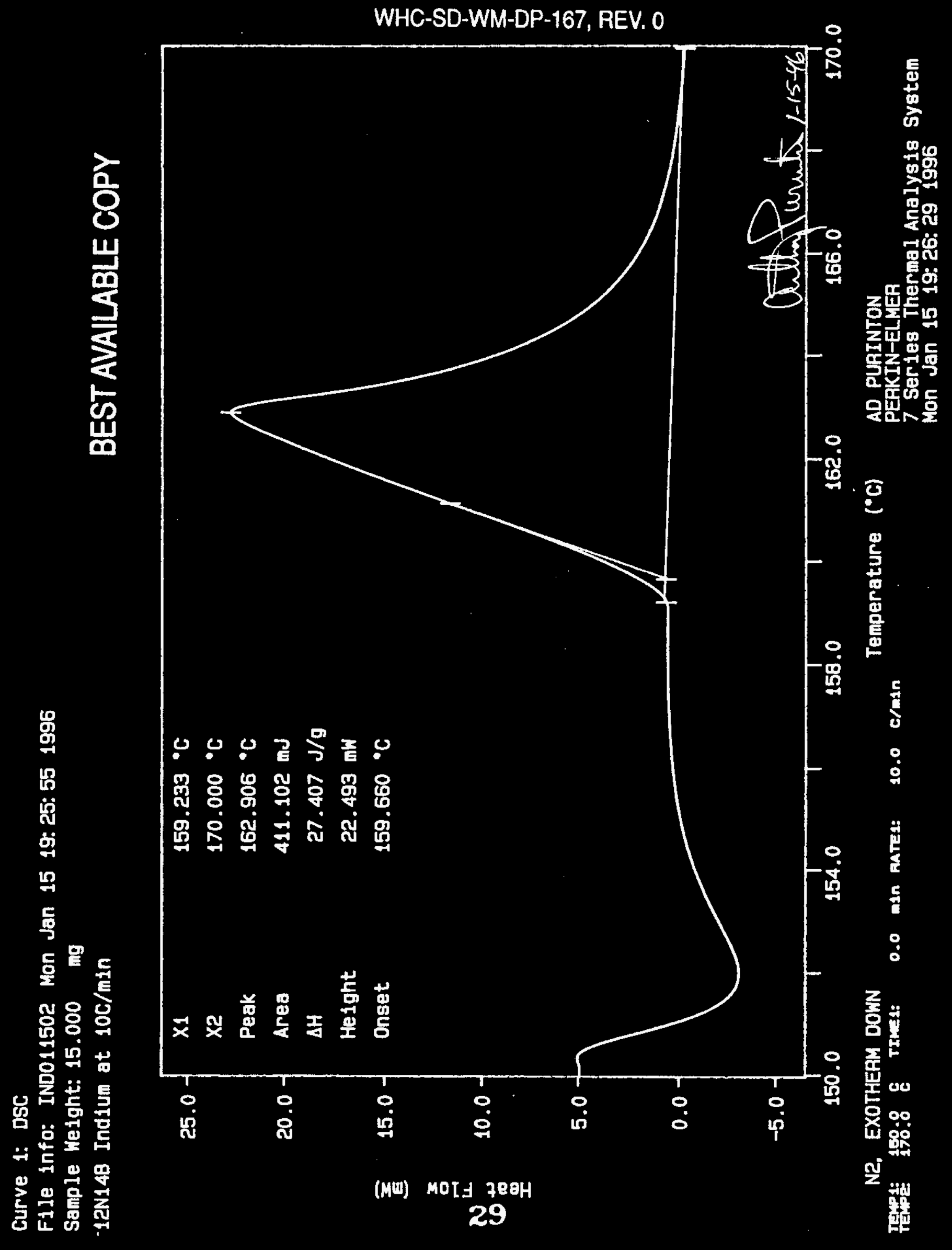


Curve 1: DSC

F1le info: SAM011506 Mon Jan 15 20: 25: 051996

Sample Weight: 42.740 ing

S96T000116 SAM

BEST AVAILABLE COPY

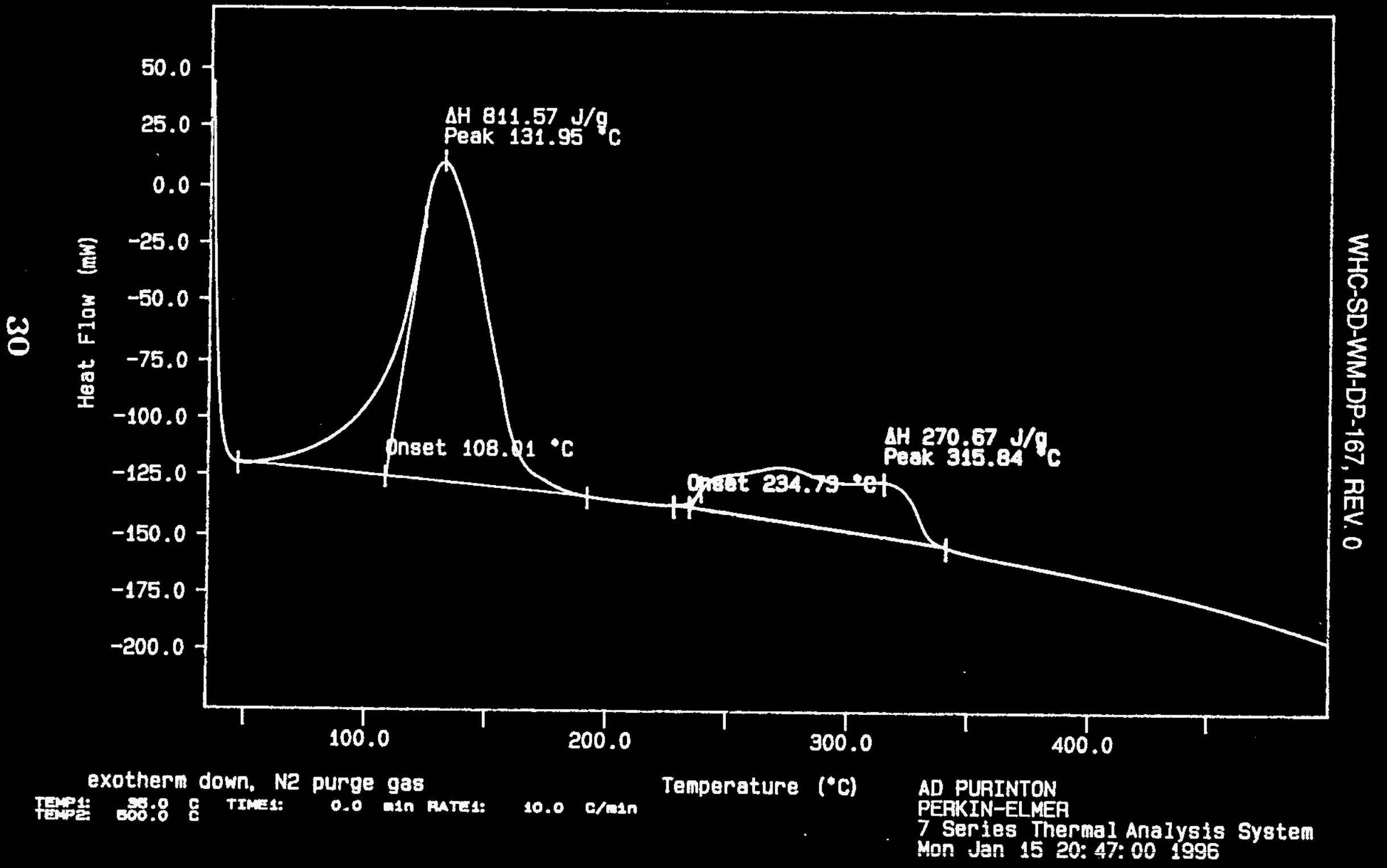




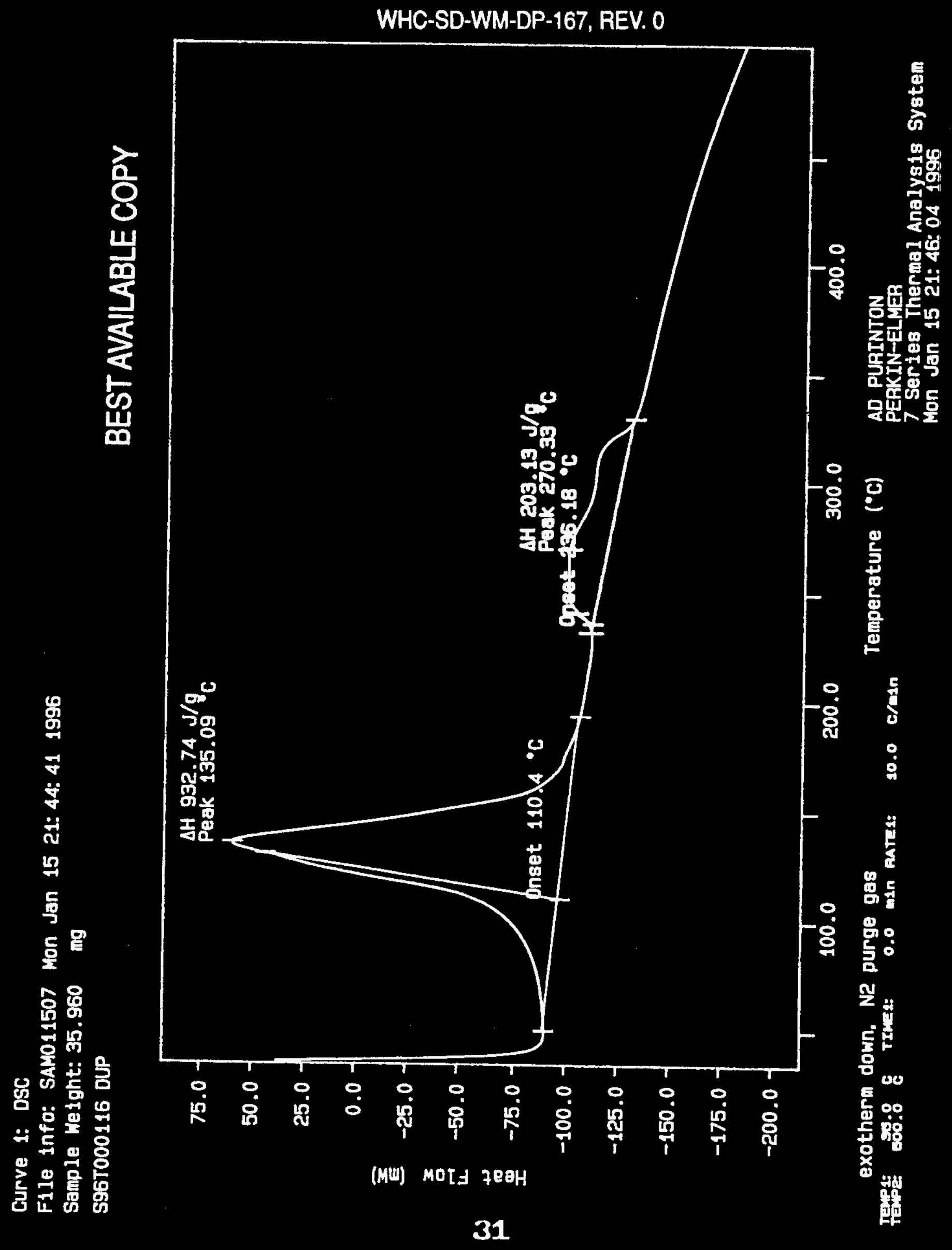




Analyst: Instrument: DSC01 Book \#-

Method: LA-514-113 Rev/Mod

Worklist Comment: Dry DSC for BX-106. bdv

\begin{tabular}{|c|c|c|c|c|c|c|c|c|c|c|}
\hline GROUP & PROJECT & S TYPE & SAMPLE\# & RA & WEST $\ldots$ & MATRIX & ACTUAL & FOUND & DL & UNIT \\
\hline 96000007 & $B X-106(A)$ & 1 SAMPLE & S96t000112 & 0 & DSC-02 & SOLID & $N / A$ & & & Joules/g Dry \\
\hline 96000007 & $8 x-106$ (A) & 2 DUP & 5961000112 & 0 & DSC-02 & SOLID & & & $\mathbf{n} / \mathbf{A}$ & Joules/g Dry \\
\hline 96000008 & $B X-106(A)$ & 3 SAMPLE & S96T000116 & 0 & DSC-02 & SOLID & $N / A$ & & & Joules/g Dry \\
\hline 96000008 & $B x-106$ (A) & 4 DUP & $596 r 000116$ & 0 & DSC-02 & SOLIO & 10 & & $N / A$ & Joules/s Dry \\
\hline
\end{tabular}

Data entered + verified by Final page for worklist \# $\mathbf{5 0 9 0}$

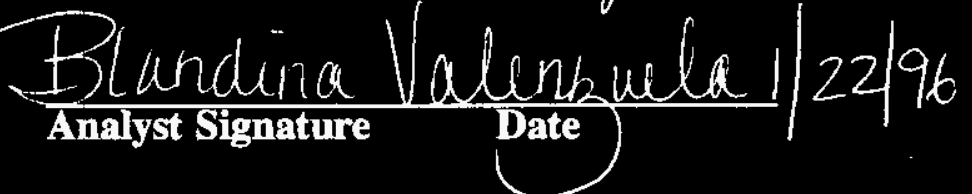

Analyst Signature Date

Data Entry Comments:

Units shown for QC (SPK \& STD) may not reflect the actual units. $D L=$ Detection Limit, $S=$ Worklist Slot Number, $\boldsymbol{R}=$ Replicate Number, $A=$ Aliquot Code. 


\section{LABCORE Data Entry Template for Worklist\#}

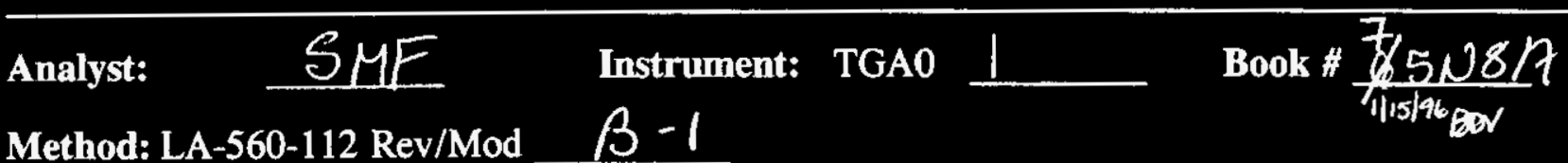

Worklist Comment: Please run BX-106 TGAs under N2. bdv

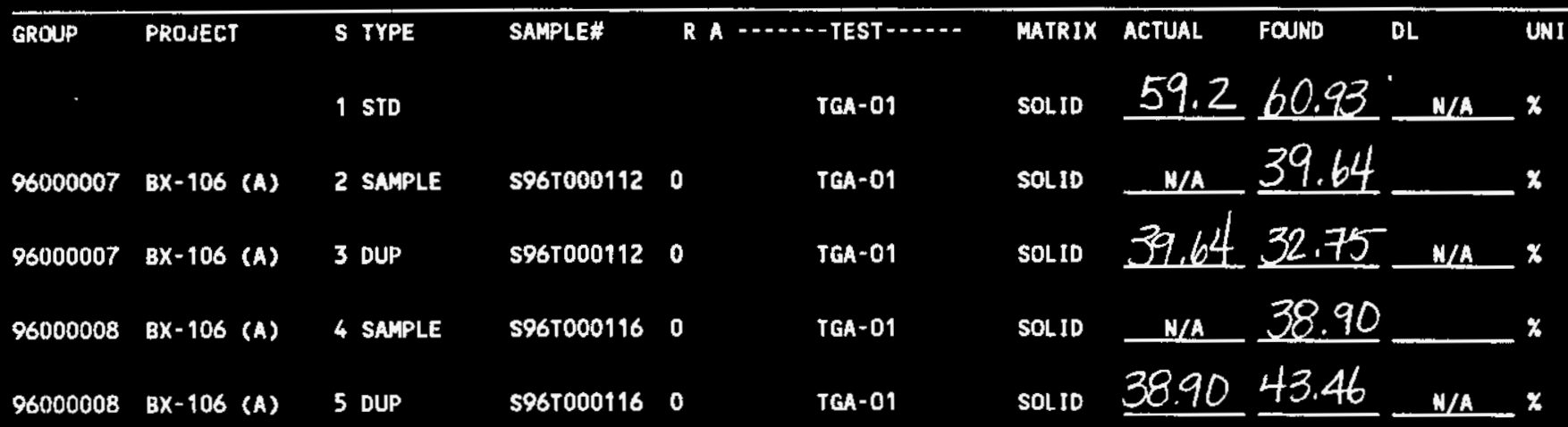

Final page for worklist \#

4847

Susi to Dutton 1-14-96

Analyst Signature Date

Verifud by Blandina Valchizula

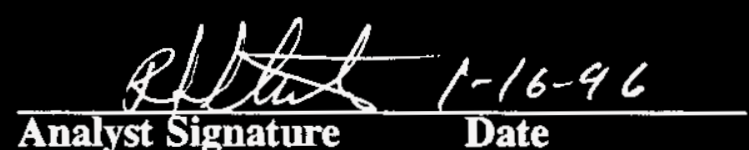

Analyst Signature Date

5967000112

Data Entry Comments: S950, produced a second weight loss step of $9.40 \%$ at epproximately $260^{\circ} \mathrm{C}$

S96T000116 pnoduced a second weight loss step of $9.13 \%$ at approximately $260 \%$

Units shown for QC (SPK \& STD) may not reflect the actual units. $D L=$ Detection Limit, $S=$ Worklist Slot Number,

$R=$ Replicate Number, $A=$ Aliquot Code. 


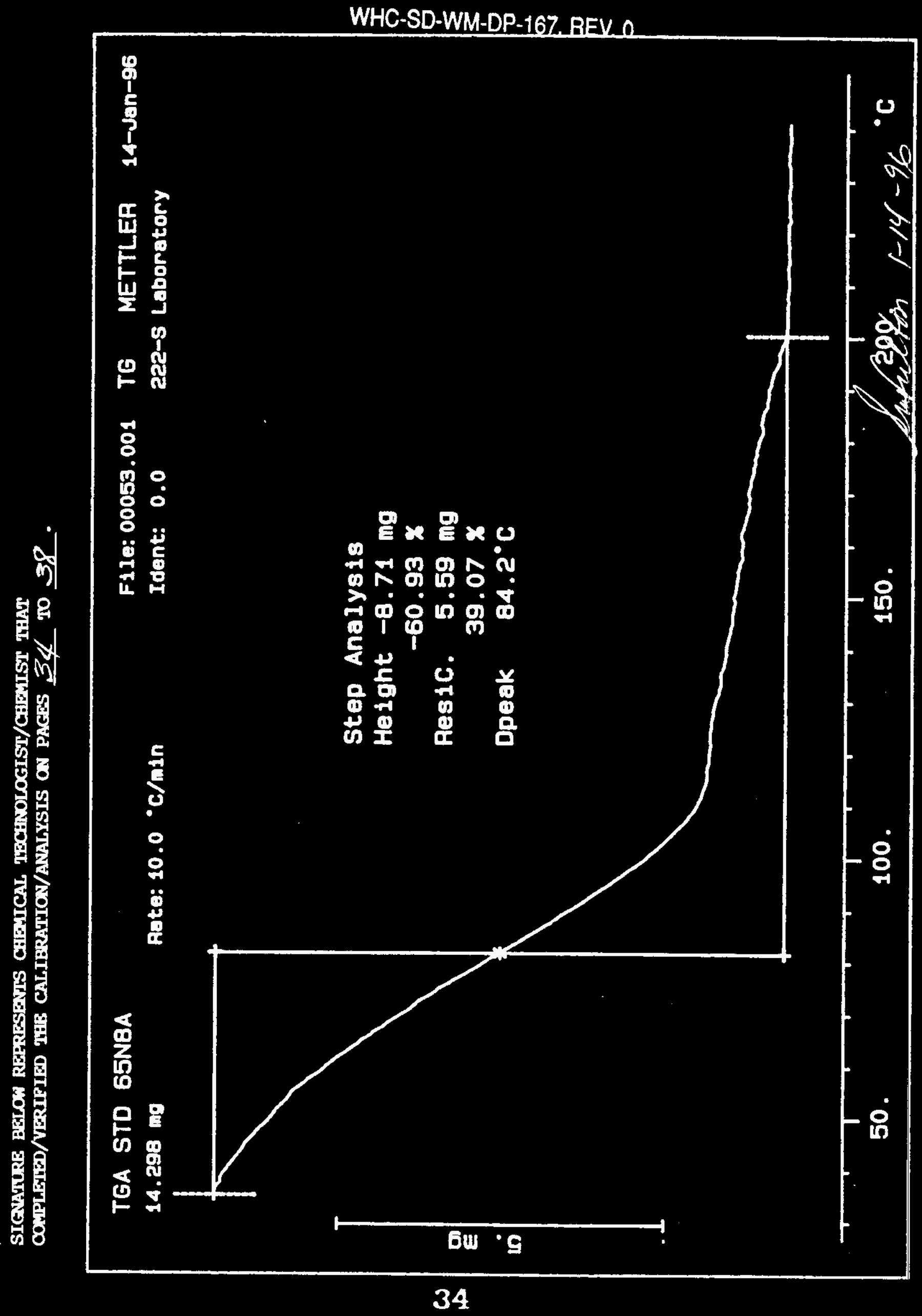




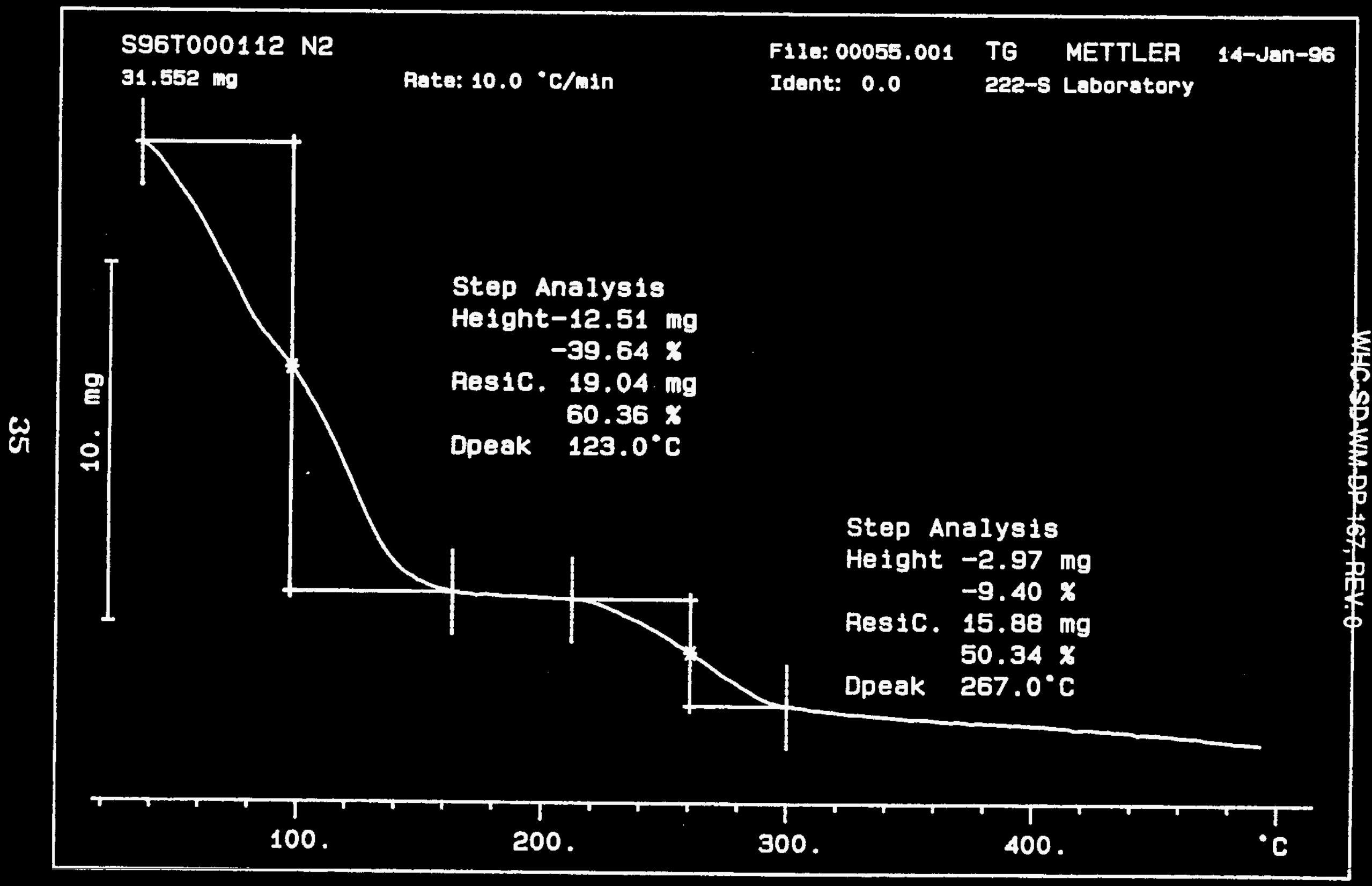




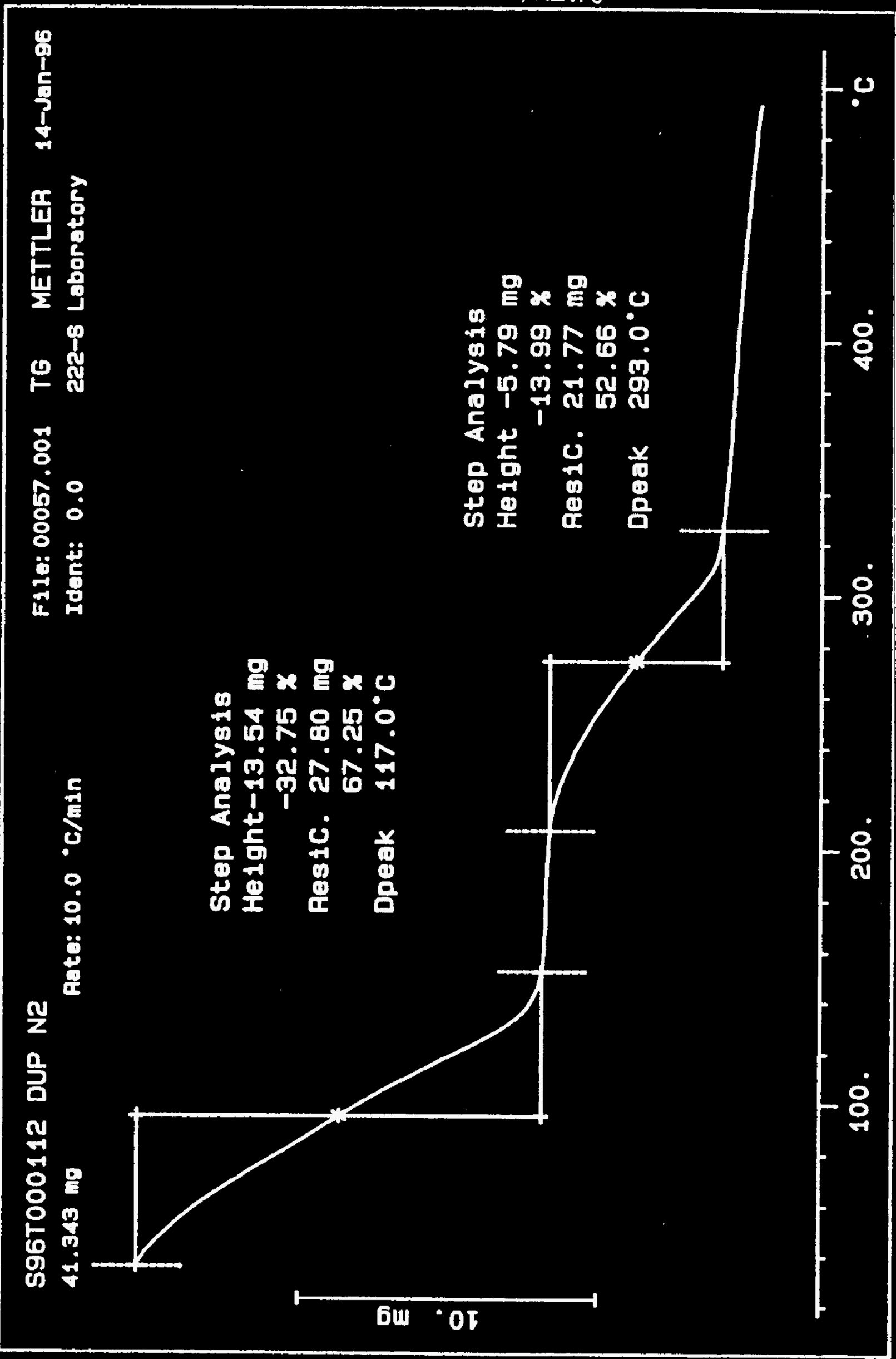


WHC-SD-WM-DP-167, REV. 0

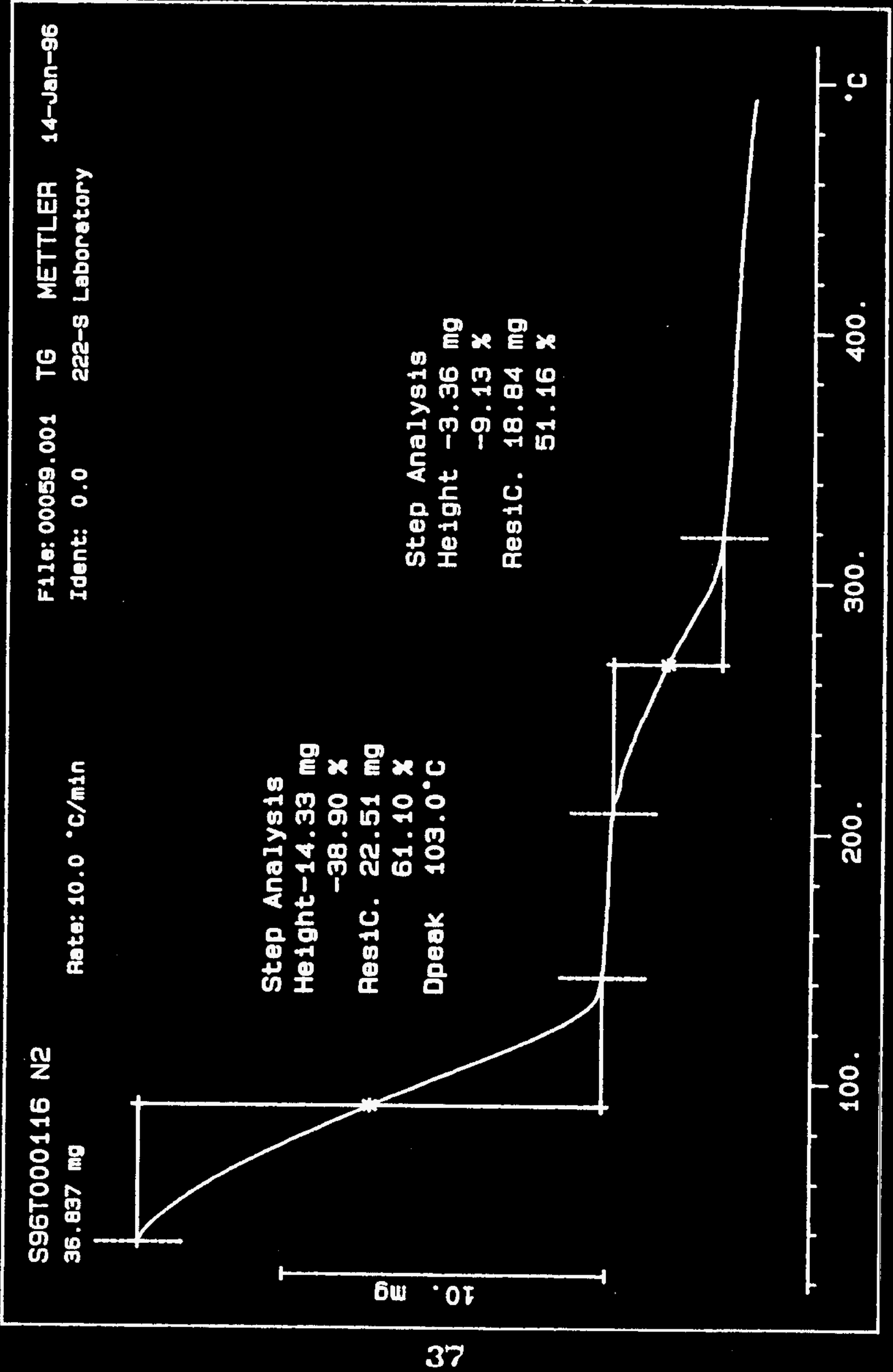




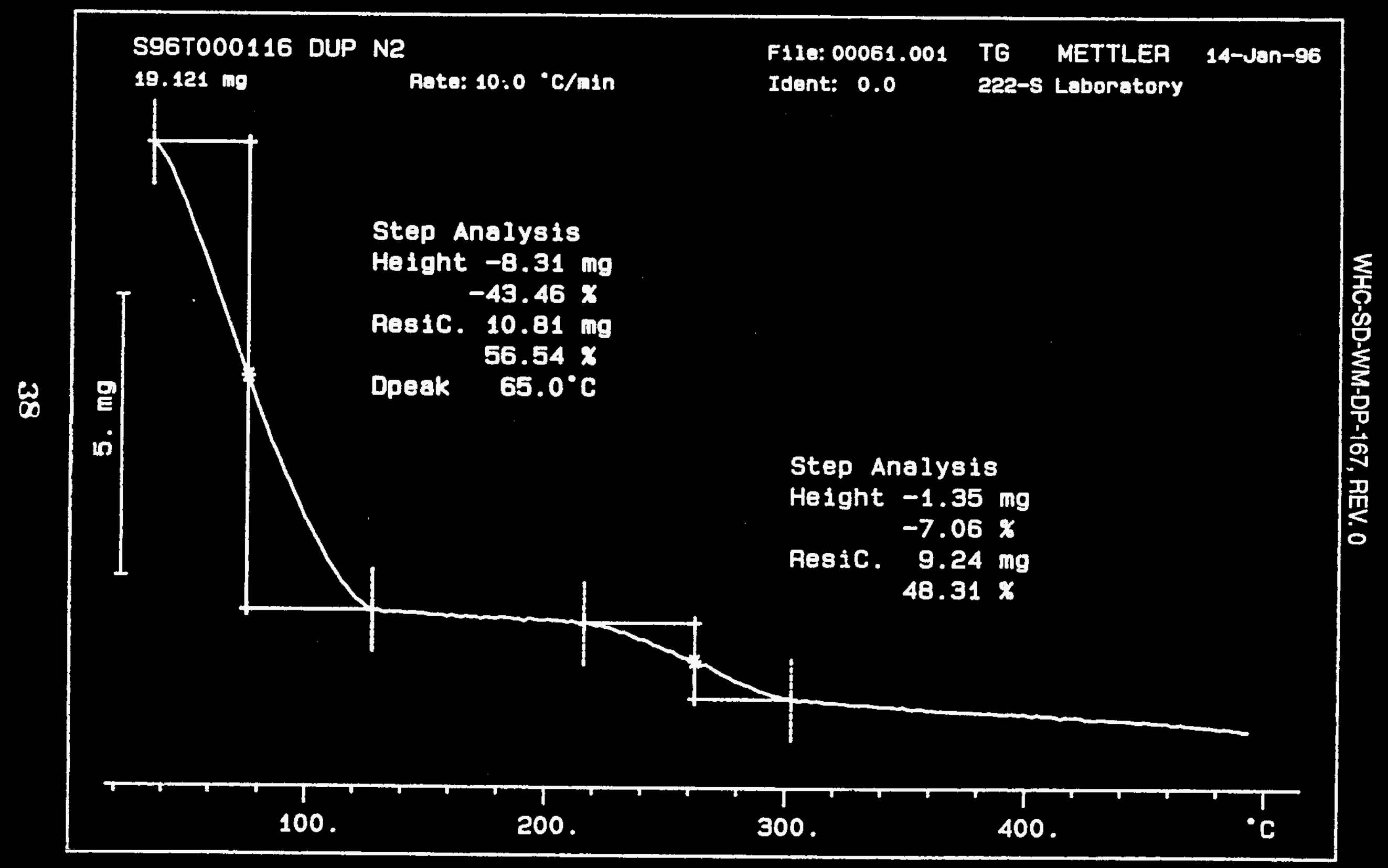


WHC-SD-WM-DP-167, REV. 0

RADIOCHEMICAL ANALYSES

39 


\section{LABCORE Data Entry Template for Worklist\# 4862}

Analyst: $\quad$ DPB Instrument: $\mathrm{AB} 00 \quad 14 \quad$ Book\# $14 \mathrm{kSE}$

Method: LA-508-101 Rev/Mod $\AA-2$

Worklist Comment: Determine sample size using Ludlum. Use . $100 \mathrm{~mL}$ A-SPK. SLF

\begin{tabular}{|c|c|c|c|c|c|c|c|c|}
\hline $\mathbf{s}$ & Type & Sample* & $\mathbf{R}$ & $\bar{A}$ & Test & Matrix & Group\# & Project \\
\hline 1 & STD & & & & QALPHA01 & SOLID & & \\
\hline 2 & BLNK-PREP & & & & QALPHAO1 & SOLID & & \\
\hline 3 & BLNK/BKG & & & & CALPHAO1 & SOLID & & \\
\hline 4 & SAMPLE & $\begin{array}{c}\text { S96T000113 } \\
\text { Analytes Reque }\end{array}$ & $\begin{array}{l}0 \\
\text { est }\end{array}$ & $\begin{array}{l}\text { E } \\
\text { ted: }\end{array}$ & $\begin{array}{l}\text { CALPHAO1 } \\
\text { : ALPHA01 }\end{array}$ & $\begin{array}{l}\text { SOLID } \\
\text {, ALPHAO1E }\end{array}$ & 96000007 & $\mathrm{BX}-106$ (A) \\
\hline 5 & DUP & s96T000113 & 0 & $F$ & CALPHAO 1 & SOLID & & \\
\hline 6 & SPK & s96T000113 & 0 & $\mathbf{F}$ & QALPHAO1 & SOLID & & \\
\hline 7 & SAMPLE & $\begin{array}{c}\text { S96T000117 } \\
\text { Analytes Reque }\end{array}$ & $\begin{array}{l}0 \\
\text { est }\end{array}$ & $\begin{array}{l}\text { F } \\
\text { ted: }\end{array}$ & $\begin{array}{l}\text { QALPHAO1 } \\
: \text { ALPHAO1 }\end{array}$ & $\begin{array}{l}\text { SOLID } \\
\text {, ALPHA01E }\end{array}$ & 96000008 & $\mathrm{BX}-106$ (A) \\
\hline 8 & DUP & $596 T 000117$ & 0 & $F$ & QALPHAO 1 & SOLID & & \\
\hline 9 & SPK & s96T000117 & 0 & F & @ALPHAO 1 & SOLID & & \\
\hline
\end{tabular}

\section{Final page for worklist \# 4862}
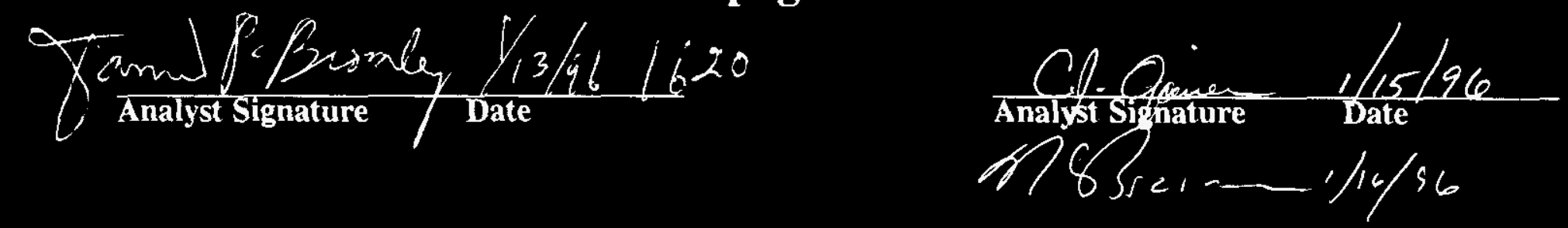

Data Entry Comments:

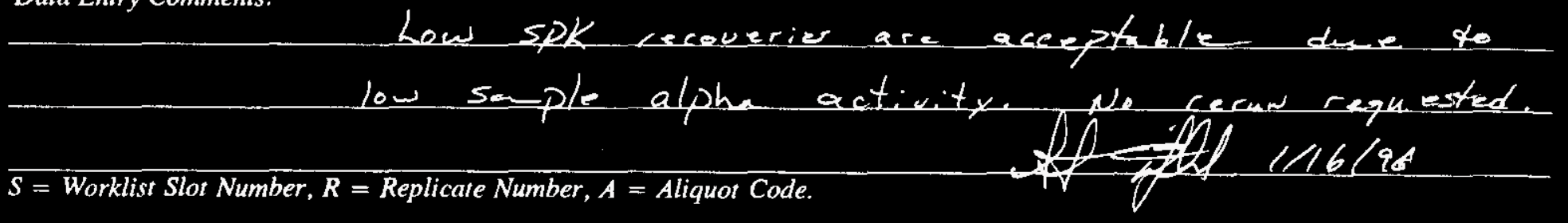




\section{LABCORE Completed Worklist Report for Worklist\# 4862}

Analyst: dpb

Instrument: $\mathrm{AB} 14$

Book"\#

Method: Rev/Mod

Worklist Comment: Determine sample size using Ludlum. Use $100 \mathrm{~mL}$ A-SPK. SLF

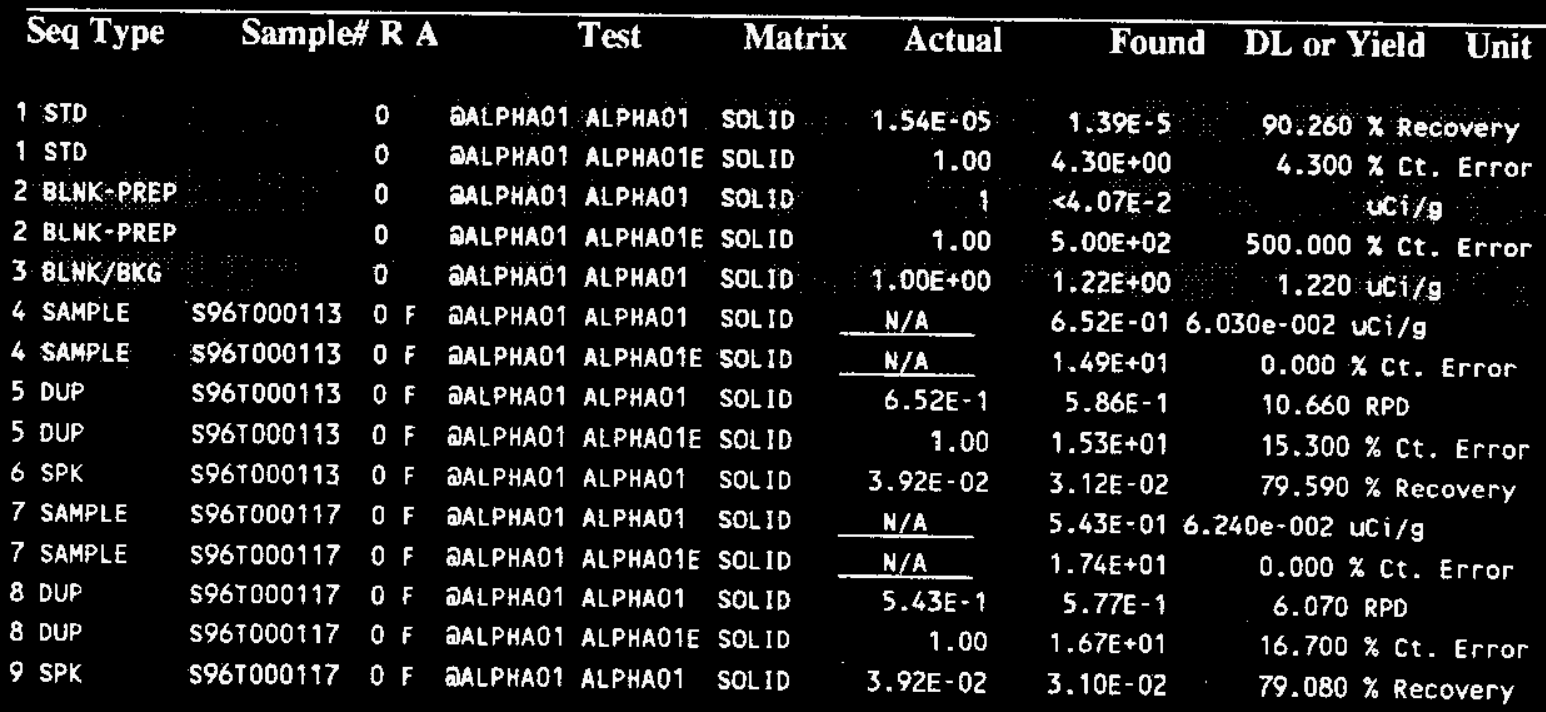

\section{Final page for worklist\# 4862}

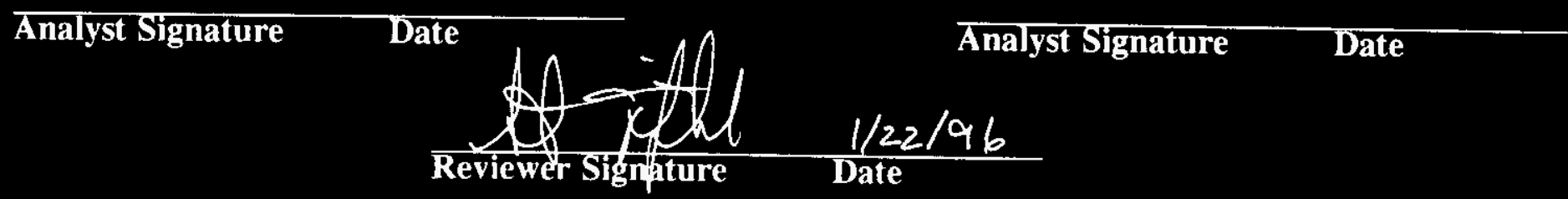


WHC-SD-WM-DP-167, REV. 0

WORKBOOK PAGE: STD1

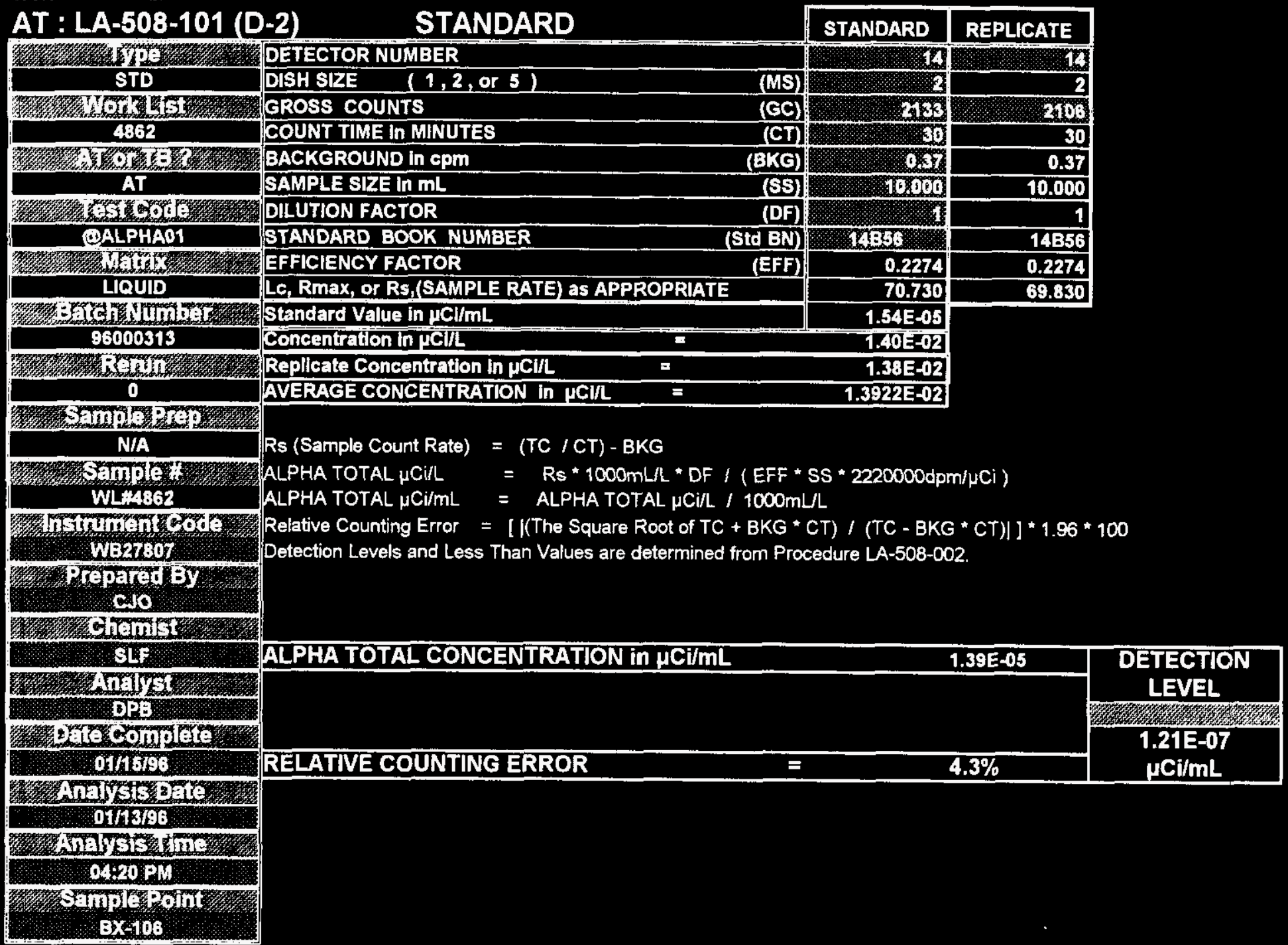

\begin{tabular}{|c|c|c|}
\hline Analyst: & $\mathrm{CJO}$ & Date: 15-Jan-96 \\
\hline Signature of Chemist: & & Date: $1 / 16 / 96$ \\
\hline
\end{tabular}


WORKBOOK PAGE: BLANK2

\begin{tabular}{|c|c|c|c|c|}
\hline$A T: L A-508-10$ & SOLIDS & BLNK-PREP & REPLICATE & \\
\hline Syope & DETECTOR NUMBER & 84 & 14 & \\
\hline BLNK-PREP & DISH SIZE $\quad(1,2$, or 5$)$ & 2 & 2 & \\
\hline Wh & GROSS COUNTS & 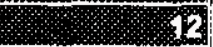 & 15 & \\
\hline 4862 & COUNT TIME in MINUTES & (30. & 30 & \\
\hline 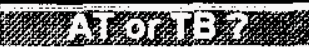 & BACKGROUND in cPm & 0.7 & 0.37 & \\
\hline AT & SAMPLE SIZE in $\mathrm{mL}$ & $00 \mathrm{sob}$ & 0.100 & \\
\hline 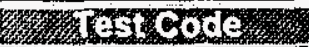 & DILUTION FACTOR & 61 & 11. & \\
\hline OALPHAOI & DIGEST GRAMS of SOLIDS $/ \mathrm{L}$ & 2.1992 & 2.1992 & \\
\hline (x) & EFFICIENCY FACTOR & 0.2274 & 0.2274 & \\
\hline $\mathbf{S O L I D}$ & LC, Rmax, or Rs,(SAMPLE RATE) as APPROPRIATE & 0.294 & 0.411 & \\
\hline 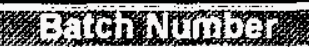 & & & & \\
\hline 96000313 & Blank concentration in uCI/g & $292=02$ & & \\
\hline 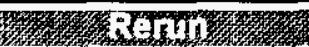 & Replicate Concentration in $\mu \mathrm{Cl} / \mathrm{g}$ & 4.07E-02 & & \\
\hline 0 & Maximum Concentration in $\mu \mathrm{CI} / \mathrm{g}$ & $4.0720 E-02$ & & \\
\hline 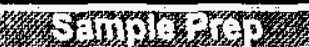 & & & & \\
\hline N/A & Rs (Sample Count Rate) $=(T C / C T)-B K G$ & & & \\
\hline SToly & $=R s * 1000 \mathrm{~mL} / \mathrm{L} * \mathrm{DF} /(\mathrm{EFF} * \mathrm{SS}$ & $\mathrm{Dg} / \mathrm{L} * 2220000$ & $\mathrm{dpm} / \mu \mathrm{Ci})$ & \\
\hline WL:4862 & & & & \\
\hline 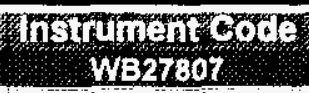 & $\begin{array}{l}\text { Relative Counting Error }=\text { [ I (The Square Root of TC + BKG * } \\
\text { Detection Levels and Less Than Values are determined from Pr }\end{array}$ & $\begin{array}{l}\text { CT) } / \text { (TC - BKC } \\
\text { cedure LA-508-0 }\end{array}$ & $\begin{array}{l}* \mathrm{CT}) \mid] * 1.96 * \\
02 .\end{array}$ & \\
\hline (n) & & & & \\
\hline 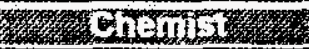 & $\checkmark$ RESULT & & & \\
\hline SLF & ALPHA TOTAL & & 4.07E-02 & DETECTION \\
\hline $3 x+1265$ & & & & LEVEL \\
\hline DPB & LESS THAN Value was Determined from Rmax. & & & \\
\hline moloondato & & & & $6.03 E-02$ \\
\hline 01115198 & RELATIVE COUNTING ERROR & & $500.0 \%$ & $\mathrm{\mu Ci} / \mathrm{g}$ \\
\hline Wholysis Beta & & & & \\
\hline DIT1396 & & & & \\
\hline 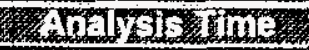 & & & & \\
\hline 0420 PM & & & & \\
\hline 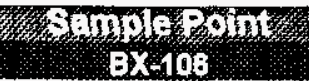 & & & & \\
\hline
\end{tabular}

\begin{tabular}{|c|c|c|}
\hline Analyst: & CJO & Date: 15 -Jan-96 \\
\hline Signature of Chemist: & & Date: $1 / 16 / 96$ \\
\hline
\end{tabular}


WHC-SD-WM-DP-167, REV. 0

WORKBOOK PAGE: SAM4

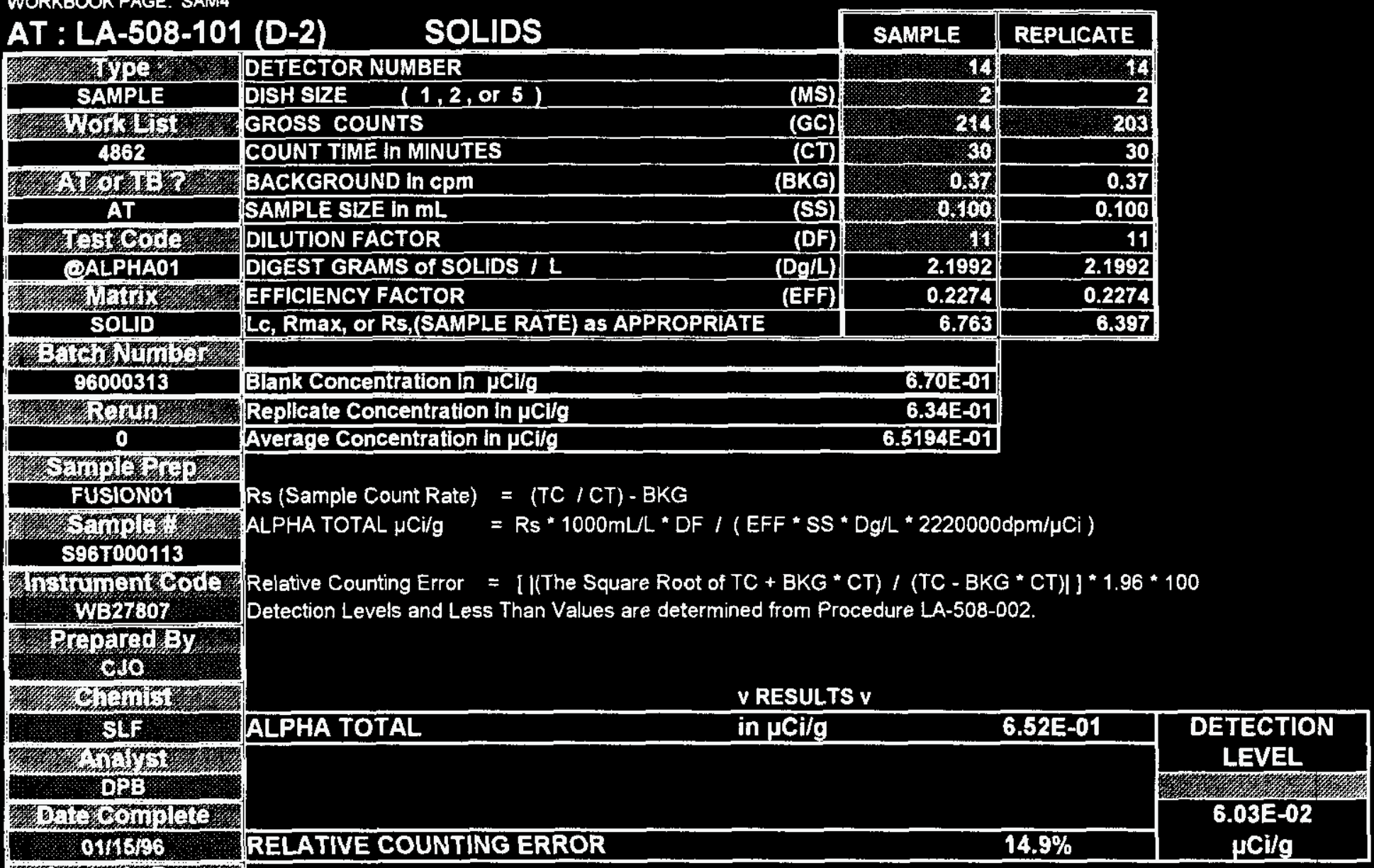

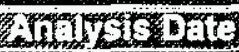
711396

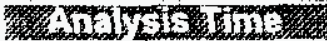
$0.420 \mathrm{PM}$

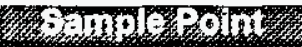
$3 \times 100$

\begin{tabular}{|c|c|c|}
\hline Analyst: & CJO & Date: $15-J a n-96$ \\
\hline Signature of Chemist: & & Date: $1 / 16 / 96$ \\
\hline
\end{tabular}


WORKBOOK PAGE: DUP5

WHC-SD-WM-DP-167, REV. 0

\begin{tabular}{|c|c|c|c|c|}
\hline & & & & \\
\hline AT : LA-508-101 & SOLIDS & DUP & REPLICATE & \\
\hline 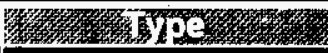 & DETECTOR NUMBER & 64 & 14 & \\
\hline DUP & DISH SIZE $\quad(1,2$, or 5$)$ & 2 & 2 & \\
\hline WOWOU W & GROSS COUNTS & 89 & 200 & \\
\hline 4862 & COUNT TIME In MINUTES & 301 & 30 & \\
\hline 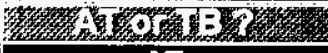 & BACKGROUND in CPm & 0.20 & 0.37 & \\
\hline AT & SAMPLE SIZE in $\mathrm{mL}$ & 0,051 & 0.100 & \\
\hline 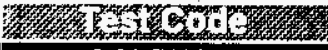 & DILUTION FACTOR & (1) & 11 & \\
\hline OALPHAO1 & DIGEST GRAMS of SOLIDS $I L$ & 2.3032 & 2.3032 & \\
\hline 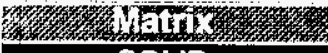 & EFFICIENCY FACTOR & 0.2274 & 0.2274 & \\
\hline SOLID & LC, Rmax, or Rs,(SAMPLE RATE) as APPROPRIATE & 6.097 & 6.297. & \\
\hline 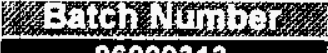 & & & & \\
\hline$\frac{96000313}{258}$ & $\begin{array}{l}\text { Blank Concentration in } \mu \mathrm{Cl} / \mathrm{g} \\
\text { Replicate Concentration in } \mu \mathrm{Cl} / \mathrm{g}\end{array}$ & $\begin{array}{l}\text { 5.77E-011 } \\
5.96 E-01\end{array}$ & & \\
\hline 0 & Average Concentration in $\mu \mathrm{C} / \mathrm{g}$ & 5.8624E-01 & & \\
\hline SLINDL & & & & \\
\hline FUSIONO1 & Rs (Sample Count Rate) $=(\mathrm{TC} / \mathrm{CT})-\mathrm{BKG}$ & & & \\
\hline S96T000113 & ALPHA TOTAL $\mu \mathrm{Ci} / \mathrm{g}=\mathrm{Rs} * 1000 \mathrm{mLL}$ * DF / (EFF * SS * & $D g / L * 2220000$ & $\mathrm{dpm} / \mu \mathrm{Ci}$ ) & \\
\hline WB27607. & $\begin{array}{l}\text { Relative Counting Error }=[\mid(T \text { The Square Root of TC }+ \text { BKG * } \\
\text { Detection Levels and Less Than Values are determined from Pro }\end{array}$ & $\begin{array}{l}\text { T) } / \text { (TC - BKG } \\
\text { edure LA-508-0 }\end{array}$ & $\begin{array}{l}\left.\left.{ }^{*} \mathrm{CT}\right) \mid\right] * 1.96 \text { * } \\
02 .\end{array}$ & \\
\hline Promer & & & & \\
\hline XX15ill & V RESULTS & & & \\
\hline SLF & ALPHA TOTAL & & 5.86E-01 & DETECTION \\
\hline (8. & & & & LEVEL \\
\hline 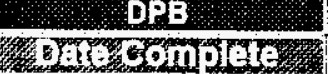 & & & & 5.76E-02 \\
\hline $01145 \% 6$ & RELATIVE COUNTING ERROR & & $15.3 \%$ & $\mathrm{uCi} / \mathrm{g}$ \\
\hline 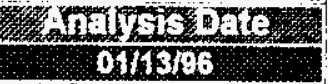 & & & & \\
\hline 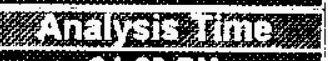 & & & & \\
\hline 04:20PM & & & & \\
\hline $0 \times 103$ & & & & \\
\hline
\end{tabular}

\begin{tabular}{|c|c|c|}
\hline Analyst: & $\mathrm{CJO}$ & Date: 15-Jan-96 \\
\hline Signature of Chemist: & SLF & Date: $1 / 16 / c$ \\
\hline
\end{tabular}




\section{WHC-SD-WM-DP-167, REV. 0}

WORKBOOK PAGE: SPIKE6

\begin{tabular}{|c|c|c|c|}
\hline \multirow{2}{*}{\multicolumn{2}{|c|}{ AT : LA-508-101 (D-2) SPIKED SAMPLE }} & \multirow[b]{2}{*}{ SPIKE } & \multirow{2}{*}{ REPLICATE } \\
\hline & & & \\
\hline 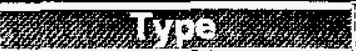 & DETECTOR NUMBER & 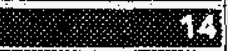 & 14 \\
\hline SPK & 1,2, or 5 & 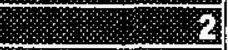 & 2 \\
\hline 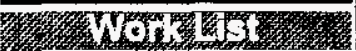 & TOTAL COUNTS & 50012 & 46991 \\
\hline 4862 & COUNT TIME in MINUTES & 30 & 30 \\
\hline DTSOSEY & BACKGROUND in cpm & OOS & 0.37 \\
\hline AT & SAMPLE VOLUME in $\mathrm{mL}$ & o.100 & 0.100 \\
\hline 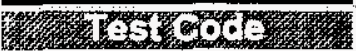 & SAMPLE DILUTION FACTOR (Spiked Vial) & 1) & 11 \\
\hline (1)ALPHAO1 & DIGEST GRAMS of SOLIDS $/ L$ & 2.1992 & 2.1992 \\
\hline $\mathrm{MLWT}$ & SPIKE VOLUME in $\mathrm{mL}$ & 0.100 & 0.100 \\
\hline SOLID & SPIKE DILUTION FACTOR & 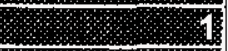 & 1 \\
\hline 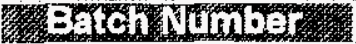 & SPIKE BOOK NUMBER & 110845 & $119 B 43$ \\
\hline 96000313 & SPIKE VALUE in $\mu \mathrm{CI} / \mathrm{mL}$ & 3.9211E-02 & $3.9211 E-02$ \\
\hline 7. & INSTRUMENT EFFICIENCY FACTOR & 0.2274 & 0.2274 \\
\hline 0 & SAMPLE + SPIKE $\mu \mathrm{Ci} / \mathrm{g}$ & $1.62 E+02$ & $1.52 E+02$ \\
\hline Stmperare & AVERAGE or MAXIMUM NCi/g in SAMPLE & $6.5194 E-01$ & \\
\hline FUSIONO1 & & & \\
\hline 3mon 15 & & & \\
\hline S96T000113 & Rs (Sample Count Rate) $=(\mathrm{TC} / \mathrm{CT})-$ BKG & & \\
\hline Wetrument code & $\begin{array}{l}\text { SAMPLE + SPIKE } \mu \text { Ci/g }=\text { Rs * 1000mLL * DF / EFF * SS } \\
\text { QC ACTUAL = SVal }\end{array}$ & * Dg/L *22200 & $\mathrm{dem} / \mu \mathrm{Ci})$ \\
\hline Prop & $\begin{array}{l}\text { QC FOUND }=\left(\left((S+S \mu \mathrm{Ci} / g-S A M P L E \mu C i / g) *\left(\left(S D F /\left(S V o{ }^{*}\right.\right.\right.\right.\right. \\
\text { PERCENT SPIKE RECOVERY }=(Q C \text { FOUND } / \text { QC ACTUAL })\end{array}$ & $\begin{array}{l}1000) /(D F / S S / D \\
100\end{array}$ & $g(L))))$ \\
\hline Chemis & & & \\
\hline StF & & & \\
\hline 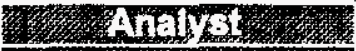 & & & \\
\hline DPB & & & \\
\hline 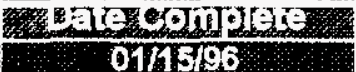 & & & \\
\hline 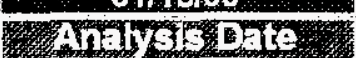 & & & \\
\hline $01 / 43196$ & QC ACTUAL & $3.92 E-02$ & \\
\hline ans & QC FOUND & 3.12E-02 & \\
\hline $04830 \mathrm{PM}$ & AVG. PERCENT SPIKE RECOVERY & $79.7 \%$ & \\
\hline 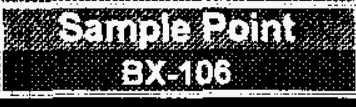 & & & \\
\hline
\end{tabular}

\begin{tabular}{|c|c|c|}
\hline Analyst: & CJO & Date: $15-J a n-96$ \\
\hline Signature of Chemist: & SLF & Date: $1 / 16 / 96$ \\
\hline
\end{tabular}


WORKBOOK PAGE: SAM7

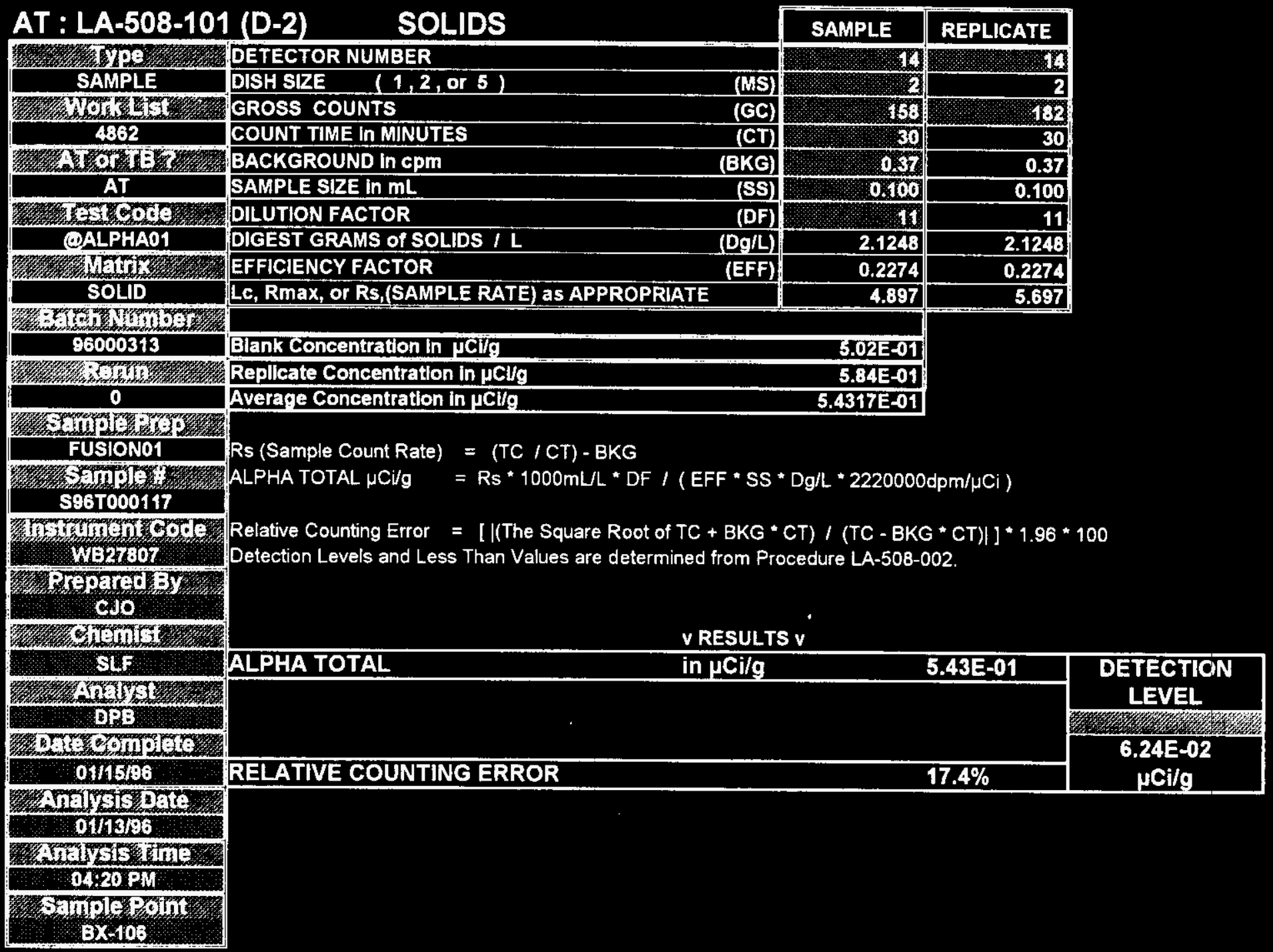

\begin{tabular}{|c|c|c|}
\hline Analyst: & CJO & Date: $15-J a n-96$ \\
\hline Signature of Chemist: & SLF & Date: $1 / 16 / 96$ \\
\hline
\end{tabular}


WORKBOOK PAGE: DUPB

\begin{tabular}{|c|c|c|c|c|}
\hline : LA-508-10 & SOLIDS & DUP & REPLICATE & \\
\hline 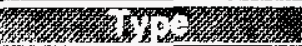 & DETECTOR NUMBER & 14 & 44 & \\
\hline DUP & DISH SIZE $\quad(1,2$, or 5$)$ & 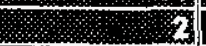 & 2 & \\
\hline MCoth & GROSS COUNTS & 78 & 189 & \\
\hline 4862 & COUNT TIME In MINUTES & $5 ; 0$ & 30 & \\
\hline 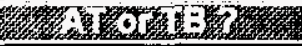 & BACKGROUND in cPm & 0.31 & 0.37 & \\
\hline$A T$ & SAMPLE SIZE $\ln \mathrm{mL}$ & 0.600 & 0.100 & \\
\hline 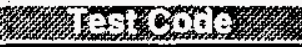 & DILUTION FACTOR & 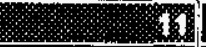 & 11. & \\
\hline PALPHAO1 & DIGEST GRAMS of SOLIDS $/ \mathrm{L}$ & 2.044 & 2.044 & \\
\hline 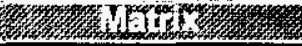 & EFFICIENCY FACTOR & 0.2274 & 0.2274 & \\
\hline SOLID & LC, Rmax, or Rs, (SAMPLE RATE) as APPROPRIATE & 5.563 & 5.263 & \\
\hline \multicolumn{5}{|l|}{ 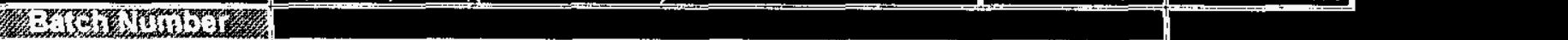 } \\
\hline 98000313 & Blank Concentration in ICl/g & 5.93E-01 & & \\
\hline 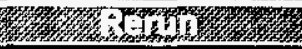 & Replicate Concentration in $\mu \mathrm{Cl} / \mathrm{g}$ & $5.61 E-01$ & & \\
\hline 0 & Average Concentration in $\mu \mathrm{Cl} / \mathrm{g}$ & $5.7708 E-01$ & & \\
\hline 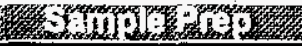 & \multirow{3}{*}{\multicolumn{4}{|c|}{$\begin{array}{l}\text { Rs (Sample Count Rate) }=(\text { TC / CT) }- \text { BKG } \\
\text { ALPHA TOTAL } \mu \mathrm{Ci} / \mathrm{g}=\mathrm{Rs} * 1000 \mathrm{~mL} / \mathrm{L} * \mathrm{DF} /(\mathrm{EFF} * \mathrm{SS} * \mathrm{Dg} / \mathrm{L} * 2220000 \mathrm{dpm} / \mu \mathrm{Ci})\end{array}$}} \\
\hline FUSIONOI & & & & \\
\hline 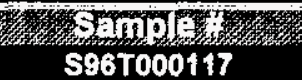 & & & & \\
\hline WB27007 & \multirow{4}{*}{\multicolumn{4}{|c|}{$\begin{array}{l}\text { Relative Counting Error }=[\text { [(The Square Root of TC + BKG *CT) / (TC - BKG *CT)] ] } 1.96 * 100 \\
\text { Detection Levels and Less Than Values are determined from Procedure LA-508-002. }\end{array}$}} \\
\hline repare $=\mathrm{y}$ & & & & \\
\hline $6 \mathrm{cso}$ & & & & \\
\hline (6) & & & & \\
\hline SLF & \multirow[t]{4}{*}{ ALPHA TOTAL } & & $5.77 E-01$ & \multirow{2}{*}{$\begin{array}{l}\text { DETECTION } \\
\text { LEVEL }\end{array}$} \\
\hline 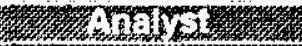 & & & & \\
\hline $\mathrm{BPB}$ & & & & 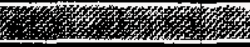 \\
\hline 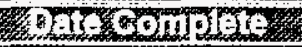 & & & & \multirow{2}{*}{$\begin{array}{l}6.49 E-02 \\
\text { pCilg }\end{array}$} \\
\hline 01115196 & RELATIVE COUNTING ERROR & & $16.7 \%$ & \\
\hline \multicolumn{5}{|l|}{ (x) } \\
\hline \multicolumn{5}{|l|}{04123106} \\
\hline \multicolumn{5}{|l|}{ 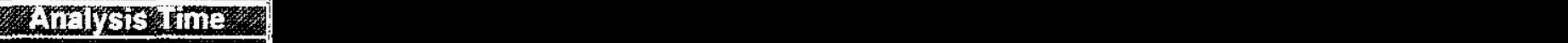 } \\
\hline (1) & & & & \\
\hline
\end{tabular}

\begin{tabular}{|c|c|c|}
\hline Analyst: & CJO & Date: 15-Jan-96 \\
\hline Signature of Chemist: & & Date: $1 / 16$ \\
\hline
\end{tabular}




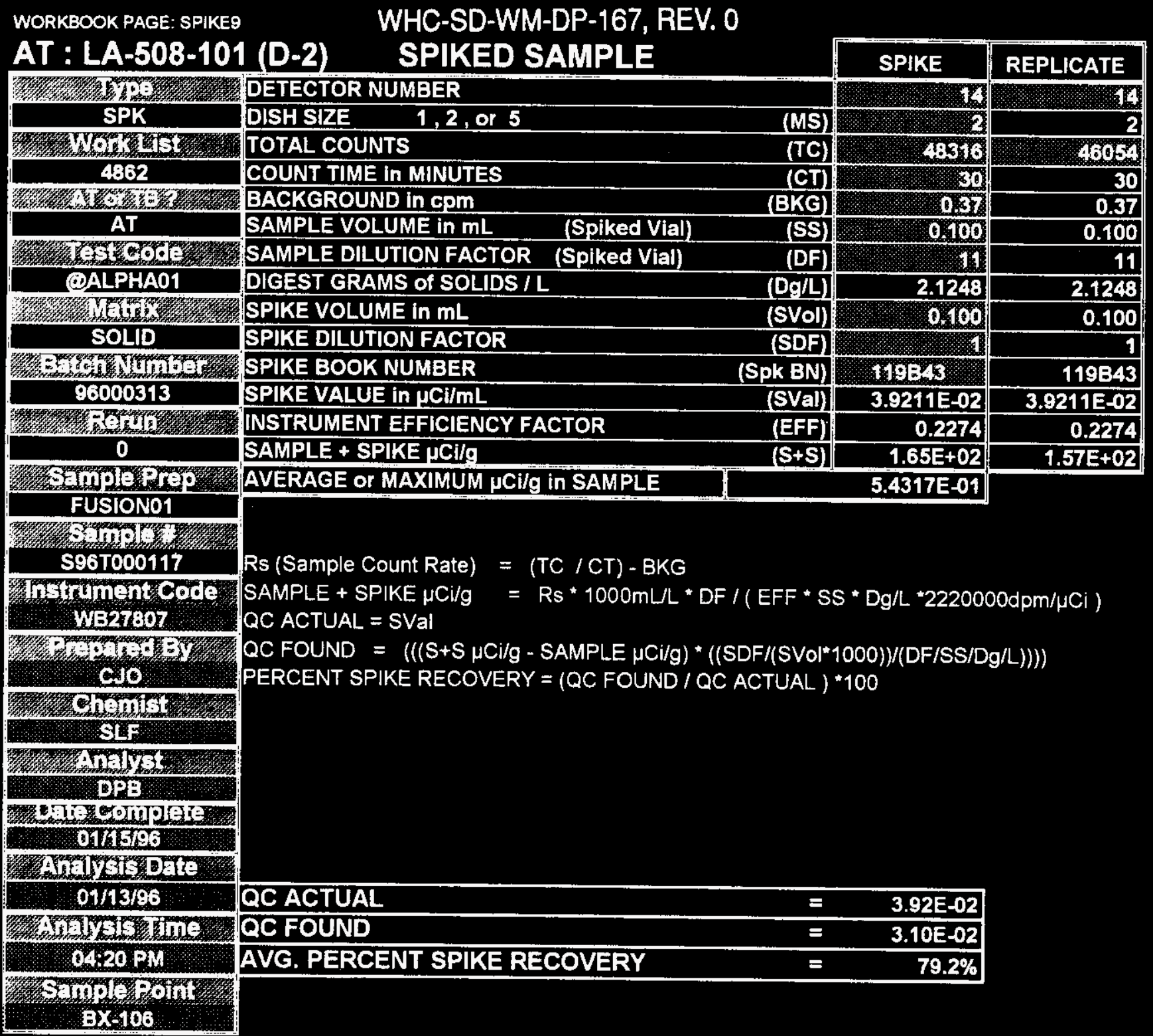

\begin{tabular}{|l|lll|}
\hline Analyst: & CJO & & Date: 15-Jan-96 \\
\hline Signature of Chemist: & SLF & Date: //L/16 \\
\hline SPIKE.WB1 Rev. 1.0
\end{tabular}




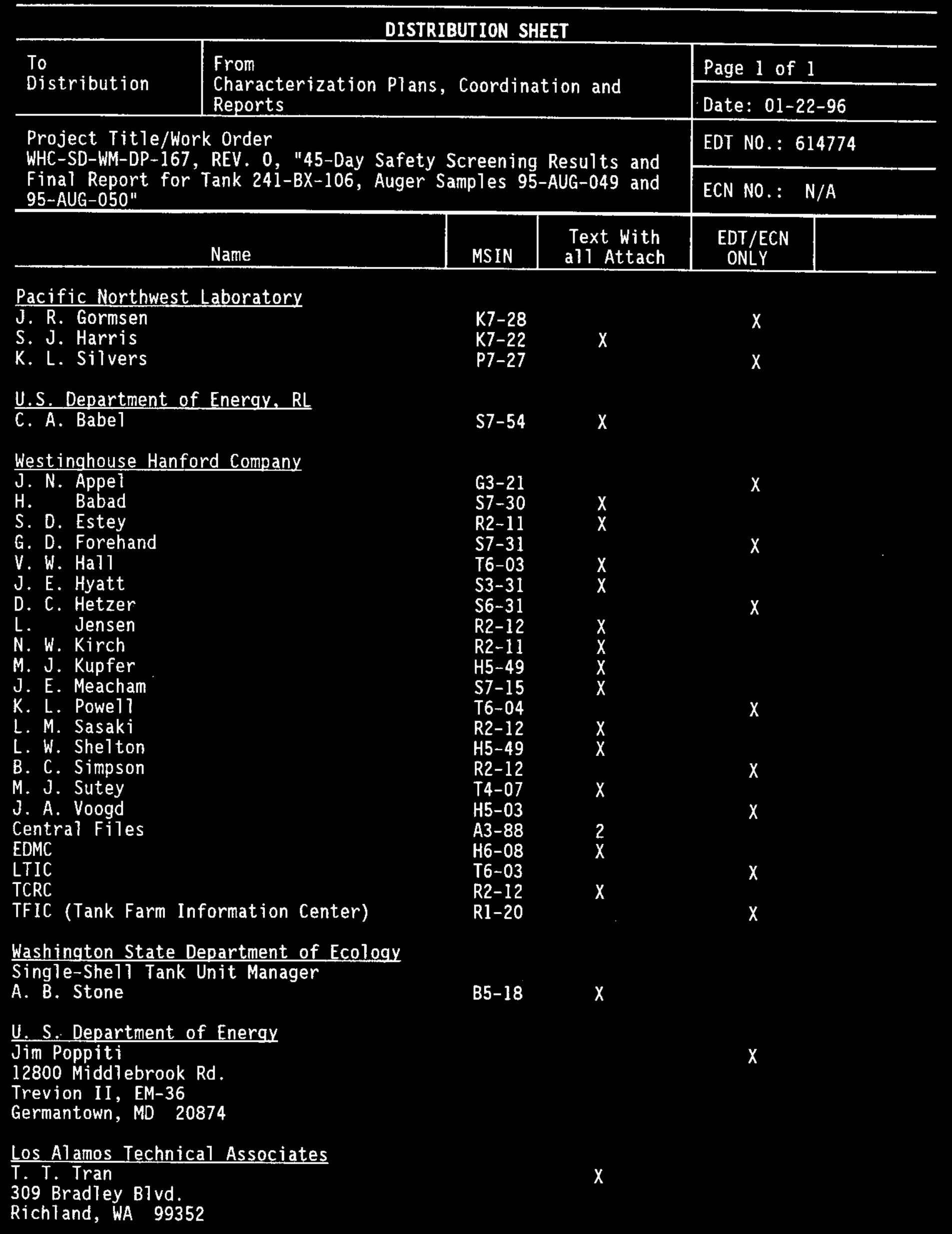

